\title{
Two and Three Body Equations in Quantum Field Models*
}

\author{
James Glimm $\star \star$ \\ Rockefeller University, New York, N.Y. 10021, USA \\ Arthur Jaffe ${ }^{\star \star \star}$ \\ Harvard University, Cambridge, Mass. 02138, USA
}

Received December 27, 1974

\begin{abstract}
In each pure phase of a $\mathscr{P}(\phi)_{2}$ quantum field model, we establish local regularity of the Green's functions and exponential decay for noncritical models. We establish the existence of twoparticle and three-particle Bethe-Salpeter kernels in the Euclidean region.
\end{abstract}

\section{Contents}

1. Introduction . . . . . . . . . . . . . . . . . . . . 293

2. $\phi^{j}$ Bounds and Schwinger Functions . . . . . . . . . . . . . . . . . . . . . . . . 296

3.1. Integration by Parts . . . . . . . . . . . . . . . . . . . . . . . . . 302

3.2. Green's Functions and Graphs . . . . . . . . . . . . . . . . . . . . . . . . . . 304

4. Regularity of the Green's Functions . . . . . . . . . . . . . . . . . . . . . . 305

5. The Support of $d q$. . . . . . . . . . . . . . . . . . . . . . . . . . . . . . . . . . . . . . . . . . . . . . . . . . . . 308

6. Decay of $\Gamma^{(2)}(x)$ and CDD Zeros . . . . . . . . . . . . . . . . . . . . . . . . . . . . 311

7. The Two-Particle Bethe-Salpeter Kernel . . . . . . . . . . . . . . . . . . . . . . 314

8. Three Particle Equations . . . . . . . . . . . . . . . . . . . . . . . . . . . . . 316

\section{Introduction}

The study of particles in weakly coupled $\mathscr{P}(\phi)_{2}$ quantum field theories was begun in $[25,26]$. The cluster expansions developed in these papers resulted in the construction of isolated one-particle states. According to the Haag-Ruelle theory, the existence of $n$-particle in and out states, and the existence of an isometric $S$-matrix, follow from the existence of isolated one particle states. The $\phi^{4}$ model, in the single phase region, has been shown to be repulsive in the sense that no even mass spectrum occurs in the two particle bound state interval $(m, 2 m)$ $[46,8,25]$. The presence of bound state mass spectrum was indicated for the $\phi^{6}-\phi^{4}$ interaction [25]. Spencer [47] and Spencer and Zirilli [48] have a more detailed analysis of the energy momentum spectrum for weak coupling, which goes up to the threshold $E \leqq 3 m-\varepsilon$, and uses the Bethe-Salpeter equation. An early version of their work motivated the present paper.

$\star$ We thank the Institut des Hautes Etudes Scientifiques for hospitality.

$\star \star$ Supported in part by the National Science Foundation under Grant MPS 74-13252.

$\star \star \star$ Supported in part by the National Science Foundation under Grant MPS 73-05037. 
In this paper we study the Bethe-Salpeter kernel in the wider class of noncritical $\mathscr{P}(\phi)_{2}$ models, and we also study three body kernels. By definition, a quantum field is called critical if its mass is zero; otherwise it is noncritical. We expect particles and a complete set of scattering states for all noncritical $\mathscr{P}(\phi)_{2}$ theories.

There are at present five (partially overlapping) methods to construct the infinite volume $\mathscr{P}(\phi)_{2}$ model. The original method [14] of compactness and convergent subsequences uses operator algebra techniques and estimates based in part on Euclidean space time localization and decoupling in the Feyman-Kac formula. This construction applies to the widest class of interactions $[14,42,43]$. The second method is the cluster expansion [25, 26], which applies in the case of weak coupling or large external field [45], and which gives the most complete information about the resulting model.

The third method $[39,28,9,44]$ in based on monotone convergence and upper bounds. The monotonicity is based on Griffiths' inequalities [12, 28], while the upper bound originates in the estimate [15]

$$
\pm \phi(h) \leqq C(h)(H+I) \text {, }
$$

see also $[27,44,9,10]$. The passage from this $\phi$-bound to estimates on Wightman functions is given in [17]. The same simple proof works for Schwinger functions. It is well known that bounds on Wightman functions imply bounds on Schwinger functions at noncoinciding points, see for example [40, 41]. In [28], a theory of conditional expectations based on the Markov property $[49,38]$ is developed to handle this point. A variant [24] of the monotonic construction replaces Dirichlet boundary conditions in (1.1) by weak coupling boundary conditions.

The main advantage of the third method is a proof of Lorentz invariance, of correlation inequalities and (for $\phi^{4}$ only) of the Lee Yang theorem. See [8, 21-24, $28,29,44,46]$ for applications.

In the fourth construction $[5,11]$, the compactness of method one is formulated in a probability framework. The fifth construction is to obtain the weakly coupled $\phi^{4}$ model by Borel summation of the perturbation series [6]. The convergence of this method is based on new estimates which require cancellation of vacuum graphs in the cluster expansion. This method gives a type of uniqueness which is particularly satisfying in view of the importance of perturbation theory in calculations.

We study the $\mathscr{P}(\phi)_{2}$ model obtained by one of these procedures. Much of Chapter 2 is an assimilation of results known in the literature for some time. Our starting point is the $\phi^{j}$ and $\pi$ bounds

$$
\begin{gathered}
\pm: \phi^{j}(h): \leqq C(h, j)(H+I) \\
\pm \pi(h) \leqq C_{\pi}(h)(H+I) .
\end{gathered}
$$

In Chapter 3 we establish the integration by parts formula for the Schwinger functions $S^{(n)}$ in each pure phase. We use this result in Chapter 4 to analyze the connected parts of the Schwinger functions (Euclidean Green's functions $G^{(n)}$ ). Let $H_{ \pm 1}$ be the Sobolev space defined by the inner product

$$
\langle f, g\rangle_{ \pm 1}=\left\langle f,\left(-\Delta+m_{0}^{2}\right)^{ \pm 1} g\right\rangle_{L_{2}} .
$$


Let $H_{ \pm 1}^{\otimes j}$ be a $j$-fold tensor product of such spaces. For noncritical $\mathscr{P}(\phi)_{2}$ theories, we prove that $G^{(n)}\left(x_{1}, \ldots, x_{n}\right)$ is the kernel of a bounded operator

$$
G^{(n)}: H_{-1}^{\otimes j} \rightarrow H_{1}^{\otimes n-j},
$$

for $0<j<n$. Here we use the $\phi^{j}$ bound, and exponential clustering, which follows from the existence of a mass gap. The stronger tree graph decay rate of the $G^{(n)}$ is known for weak coupling [6]. Since $G^{(n)}$ is translation invariant, it cannot be compact. However, we show that the restriction of $G^{(n)}$ to a subspace of definite total momentum is Hilbert-Schmidt. This is a crucial bound on the $G^{(n)}$.

An immediate corollary of this bound on $G^{(n)}$ is the fact that noncritical Schwinger functions $S^{(n)}$ for the field $\hat{\phi}=\phi-\langle\phi\rangle$ are continuous multilinear functionals on the $n$-fold product $H_{-1} \times \ldots \times H_{-1}$. This result was established for weak coupling in [20]. A second corollary is the existence of the vertex functions $\Gamma^{(n)}\left(x_{1}, \ldots, x_{n}\right)$ (direct correlation functions) for noncritical $\mathscr{P}(\phi)_{2}$ theories. The $\Gamma^{(n)}$ are bounded operators from $H_{1}^{\otimes j}$ to $H_{-1}^{\otimes n-j}, 0<j<n$, improving slightly a bound from [20].

In Chapter 6 we show that the inverse propagator $\Gamma(x)$ has an exponential decay rate equal to the CDD radius $\bar{m}$. Here $m<\bar{m} \leqq M$ where $m$ is the single particle mass and $(m, M)$ is the upper gap in the spectrum of the two point function.

In Chapter 7 we construct the 2-particle Bethe-Salpeter kernel for non-critical $\mathscr{P}(\phi)_{2}$ models. We use the compactness of the $G^{(n)}$ on constant momentum subspaces, and apply a Fredholm alternative. The exclusion of the eigenvalue zero in the Fredholm alternative is related to the following property, which we establish in Chapter 5 for a $\mathscr{P}(\phi)_{2}$ measure: If a polynomial $\mathscr{R}(\phi)$ takes the value zero almost everywhere on $\mathscr{S}^{\prime}\left(R^{2}\right)$, then $\mathscr{R}=0$, i.e. the coefficients of $\mathscr{R}$ vanish. This property can be rephrased by saying that the Euclidean $\mathscr{P}(\phi)_{2}$ measure is not supported on any algebraic variety in $\mathscr{S}^{\prime}\left(R^{2}\right)$. We also require this property for polynomials $\mathscr{R}(\phi)$ with a definite momentum. It is a consequence of integration by parts, or of the existence [11] of Euclidean momentum operators $\pi$. We remark that some time ago Bros [3] constructed the two body Bethe-Salpeter kernel in the context of the Wightman axioms, plus additional assumptions. Finally in Chapter 8, we consider three particle equations.

The two body Bethe-Salpeter kernel is a bounded operator

$$
K: H_{1}^{\otimes 2} \rightarrow H_{-1}^{\otimes 2}
$$

satisfying the integral equation

$$
R=R_{0}+R_{0} K R
$$

in the case of even $\mathscr{P}(\phi)$ models. Here $R$ and $R_{0}$ map $H_{-1}^{\otimes 2}$ into $H_{1}^{\otimes 2}$ and are defined by their kernels

$$
\begin{aligned}
R_{0}\left(x_{1}, x_{2} ; y_{1}, y_{2}\right) & =G^{(2)}\left(x_{1}, y_{1}\right) G^{(2)}\left(x_{2}, y_{2}\right)+G^{(2)}\left(x_{1}, y_{2}\right) G^{(2)}\left(x_{2}, y_{1}\right), \\
R\left(x_{1} x_{2} ; y_{1} y_{2}\right) & =G^{(4)}\left(x_{1}, x_{2}, y_{1}, y_{2}\right)+R_{0}\left(x_{1}, x_{2} ; y_{1}, y_{2}\right) .
\end{aligned}
$$

Thus

$$
K=R_{0}^{-1}-R^{-1},
$$


and formally $K$ is a sum of graphs two particle irreducible between initial and final states. The construction of $K$, however, does not have an immediate significance for the particle structure. In fact the estimates [47] which yield decay properties of $K\left(x_{1}, x_{2} ; y_{1}, y_{2}\right)$ depend on the cluster expansion, and are known only for weak coupling. The methods of Lebowitz and Penrose [31,32] from statistical mechanics given an analytic continuation of decay estimates. The Lebowitz-Penrose results pertain only to the gap between the ground state and the first excited state, and are not strong enough to give the existence of particles. The extension of the Lebowitz-Penrose result [32] to field theory is contained in [29]. It is an interesting speculation that nontrivial decay properties for $K\left(x_{1}, x_{2}, y_{1}, y_{2}\right)$ are a consequence of the existence of a mass gap. However analyticity properties in the coupling should not be a consequence of a positive mass, even in the case $\mathscr{P}=$ even, cf. [24, Fig. 2.1].

\section{2. $\phi^{j}$ Bounds and Schwinger Functions}

Let $\mathfrak{A}$ be the $C^{*}$-algebra of the $V=\infty$ limit, acting on a Hilbert space $\mathscr{H}$. By $[1], \mathfrak{U}^{\prime}=\mathscr{Z}$, the center of $\mathfrak{U}^{\prime \prime}$. The direct integral decomposition is defined with respect to $\mathscr{Z}$; we write it as

$$
\mathscr{H}=\int \mathscr{H}(\xi) d \xi
$$

so that $\xi \in \Xi$ is a label for the set $\Xi$ of phases. Let $\Omega_{\xi} \in \mathscr{H}_{\xi}$ be the vacuum and let $H_{\xi}$ be the Hamiltonian. For example, in the $\phi_{2}^{4}$ model we expect a single phase for $\lambda<\lambda_{c}$, and two phases for $\lambda>\lambda_{c}$. We expect a continuum of phases in the case of phase transitions with a continuous group of internal symmetries, so this direct integral machinery may not be too elaborate for the use we make of it. See $[25,11]$ for a further discussion of phases.

We now state our basic $\phi$ and $\pi$ bounds. Let

$$
1 \leqq j \leqq d=\operatorname{deg} \mathscr{P} \text {. }
$$

For general polynomials, all constructions $[14,11]$ assume free boundary conditions. For $\mathscr{P}=$ even + linear, we allow either free or weak coupling boundary conditions [24]. We let $V$ denote the volume of interaction; for weak coupling boundary conditions, $V$ is the length of the interval $[-b, b]$ in which the mass perturbation $\sigma \int_{-b}^{b}: \phi^{2}(x): d x$ is inserted. Note that for $V=\infty$, weak coupling and Dirichlet boundary data define the same theory, for $\mathscr{P}=$ even + linear [24].

Theorem 2.1 a ( $\phi^{j}$ and $\pi$ bounds). For $V<\infty, j \leqq d$

$$
\begin{aligned}
& \pm \phi(h) \leqq\|h\|_{1} C(H+I), \\
& \pm \pi(h) \leqq\|h\|_{2} C(H+I), \\
& \pm: \phi^{j}:(h) \leqq H+O\left(\left\|\left(|x|^{r}+1\right) h\right\|_{d / d-j}+\|h\|_{d / d-j}^{d / d-j}\right),
\end{aligned}
$$

for $h$ real and in the appropriate $L_{p}$ space, and for $r>1+(j \mid d)$. Here $C$ is a constant, independent of $h$ and $V$. In the case $j=d$, we also require $\|h\|_{\infty}$ small. 
Theorem 2.1 b ( $\phi^{j}$ bounds). For $j \leqq d, V=\infty$ and in a.e. pure phase, the bounds (2.2a) and (2.2c) are valid. Moreover for free boundary conditions

$$
\pm: \phi^{j}:(h) \leqq H+O\left(\|h\|_{1}+\|h\|_{d / d-j}^{d / d-j}\right) .
$$

Proof of Theorem 2.1a. (2.2a) was proved in [15]. The bound (2.2b) was established in $[16,17]$ for $V<\infty$, and the same methods can be used directly in the infinite volume limit. Bounds related to (2.2c) were first given in [15] and have been improved independently by Fröhlich [11] and the authors (unpublished). For weak coupling, Dirichlet, or free boundary conditions and $V<\infty$, we use the inequality

$$
\pm: \phi^{j}:(h) \leqq H+O(a)+O(1)\|h\|_{d / d-j}^{d / d-j}
$$

for support $h \subset[-a, a]$, cf. $[24,51]$. Because the vacuum energy of the : $\phi^{j}$ : perturbation is convex, and is zero for $h=0$, this yields

$$
\pm: \phi^{j}:(h) \leqq H+O(a)^{j / d}\|h\|_{d / d-j}+O(1)\|h\|_{d / d-j}^{d / d-j} .
$$

We write $h=\sum h_{n}$, suppt $h_{n} \subset[n, n+1]$, and then by convexity again,

$$
\pm: \phi^{j}:(h) \leqq H+O(1) \sum\left\|(|n|+1) h_{n}\right\|_{d / d-j}+O(1)\|h\|_{d / d-j}^{d / d-j} .
$$

Finally,

$$
\begin{aligned}
\sum\left\|(|n|+1) h_{n}\right\|_{d / d-j} & =\sum(|n|+1)^{-\alpha}\left\|(|n|+1)^{1+\alpha} h_{n}\right\|_{d / d-j} \\
& \leqq \text { const. }\left\|(|x|+1)^{1+\alpha} h\right\|_{d / d-j}
\end{aligned}
$$

for $\alpha>j / d$.

Proof of Theorem 2.1b. The transfer of the $\phi$ bound to the infinite volume limit is given in [15]. The infinite volume limit of the : $\phi^{j}$ : bounds is established in $[24]$.

There is also an abstract method for transferring an estimate of the form

$$
\pm A \leqq H+I
$$

to the infinite volume limit, and then into almost every phase [2]. This method requires an additional estimate, for example

$$
\left\|A(H+I)^{-1}\right\|+\left\|[H, A](H+I)^{-1}\right\|<\text { const. }
$$

uniform in the volume $V$. Such an estimate holds in the case $A=\phi(h), h \in \mathscr{S}\left(R^{1}\right)$, using [15, Lemma 1.1] and (2.2a)-(2.2c). Hence we obtain (2.2a), with $h \in \mathscr{S}$, in the $V=\infty$ limit and in almost every pure phase. The role of the inequality (2.6) is to provide a domain of essential self-adjointness ${ }^{1}$ for $A=\phi(h)$. Having established the bound and decomposition to pure phases for $h \in \mathscr{S}$, we pass to $h \in L_{1}$ using (2.2a). (2.2c) is a consequence of $(2.2 \mathrm{~d})$, for $V=\infty$. The bound $(2.2 \mathrm{~d})$ is given in [11].

1 The general method of using commutation estimates to establish self adjointness was introduced in [13] to prove self adjointness of space-time averaged field operators $\phi(f)$. One abstraction of this method was presented in [18], and another abstraction was presented in [15]. These results established essential self adjointness of an operator or form $A$, satisfying relative bounds with respect to a positive self adjoint operator $H$. In our present applications, we use [15: Lemma 1.1, Theorem 1.2] to establish essential self adjointness of $\phi(h)$. Other abstractions and applications have also been given by Nelson [37], McBryan [33 - 35], Faris und Lavine [7], and McBryan and Park [36]. 
In the case of weak coupling boundary conditions, the basic method relies on uniform estimates $[10,24]$ for the analytic generating functionals of the ordinary and generalized Schwinger functions. By compactness, these functionals have a limit point, as $b \rightarrow \infty$, and the limit point satisfies the same estimate. However, convergence of the Schwinger functions as $b \rightarrow \infty$ [24; see also Theorem 2.2, 2.4 below] implies that the analytic functions actually have a limit as $b \rightarrow \infty$. The Schwinger functions are constructed from the generating functionals [10] and yield the infinite volume Hilbert space [10, 40, 41]. On this Hilbert space, the bound (2.2c) holds in the sense of a norm estimate on the perturbed semigroup [24]. Further details are omitted.

Theorem 2.2. Let $h, \chi \in C_{0}^{\infty}, \int \chi d x=1,0 \leqq \chi$, and $V<\infty$, and

$$
\delta H_{\chi}=\int \prod_{i=1}^{j}(\phi * \chi)(x) h(x) d x .
$$

Then

$$
\pm \delta H_{\chi} \leqq H+O(1)
$$

uniformly as $V \rightarrow \infty$. Let $\chi_{i}=\kappa_{i} \chi(\kappa ; x), i=1,2$. Then

$$
\pm\left(\delta H_{\chi_{1}}-\delta H_{\chi_{2}}\right) \leqq H+o(1)
$$

uniformly in $V$, as $\kappa_{1}, \kappa_{2} \rightarrow \infty$. Again for $j=d$, we require $\|h\|_{\infty}$ small.

Theorem 2.2 follows as an extension of the methods of [15] and [51] for free and Dirichlet boundary conditions, respectively. It is presumably also true for weak coupling boundary conditions, but we do not require this fact.

We need to introduce certain function spaces related to the estimates of Theorem 2.1 Let

$$
\left\{\begin{array}{l}
\mathscr{L}_{1, p}=\mathscr{L}_{1}\left(R^{2}\right) \cap \mathscr{L}_{p}\left(R^{2}\right), \quad p<\infty \\
\mathscr{L}_{1, \infty, \varepsilon}=\mathscr{L}_{1}\left(R^{2}\right) \cap\left\{f:\|f\|_{\infty}<\varepsilon\right\} .
\end{array}\right.
$$

Whenever $p=\infty$, the space $\mathscr{L}_{1, \infty, \varepsilon}$ will be understood, even if the $\varepsilon$ is not explicitly included.

Before studying the quantum field on the space $\mathscr{H}(\xi)$, we require more information about its action on $\mathscr{H}$.

Theorem 2.3. The quantum field on $\mathscr{H}$, specified by a $C^{*}$ algebra construction, is unitarily equivalent to an Osterwalder-Schrader [40,41] reconstructed field. The Schwinger functions are moments of a unique measure $d q$ on $\mathscr{S}^{\prime}\left(R^{2}\right)$.

Remark. Since the covariance of the field under Lorentz transformations is not known, the corresponding imaginary time fields on $\mathscr{S}^{\prime}\left(R^{2}\right)$ will be time translation covariant, but the Euclidean invariance is not known.

Proof. We verify the axioms (A1)-(A5), B, C of Fröhlich [9] in order to obtain the imaginary time fields and the reconstructed fields. Thus we want to study the generating functions

$$
J(f)=\int e^{i \phi(f)} d q
$$


of the imaginary time measure. For $f$ piecewise constant in $t, J(f)$ has a Hamiltonian definition. If $h_{1}(x), \ldots, h_{n}(x)$ are piecewise constant values of $f(\cdot, t)$, then

$$
J(i f)=\left\langle\Omega, e^{-\left(t_{2}-t_{1}\right)\left(H+\phi\left(h_{1}\right)\right)} \ldots e^{-\left(t_{n}-t_{n-1}\right)\left(H+\phi\left(h_{n}\right)\right)} \Omega\right\rangle .
$$

For a finite space cutoff, we know that $e^{-t H}$ is positivity preserving and that the imaginary time measure on $\mathscr{S}^{\prime}\left(R^{2}\right)$ exists. Also $J(f)$ is complex analytic in $f \in \mathscr{S}\left(R^{2}\right)$, for example, and can be bounded using Theorem 2.1.

For any operator $H$, we define its vacuum energy to be $E(H)=\inf$ spectrum $H$. Let

$$
\delta E(t, f)=E(H+\phi(f(\cdot, t)))-E(H),
$$

for $f$ real. Then by Theorem 2.1,

$$
\begin{aligned}
|J(f)| & \leqq \exp -\int_{-\infty}^{\infty} \delta E(t, \operatorname{Im} f) d t \\
& \leqq \exp \text { (const. }\left(\|f\|_{d / d-1}^{d / d-1}+\int\left\|\left(|x|^{r}+1\right) f(\cdot, t)\right\|_{d / d-1} d t\right)
\end{aligned}
$$

with a constant uniform in the space cutoff. By Vitali's theorem, $J$ is complex analytic for $f \in \mathscr{L}$ where $\mathscr{L}$ is defined by the norms in (2.9). Following Fröhlich [10], we conclude that the space cutoff Schwinger $n$-point function is a bounded multilinear functional on $\mathscr{L} \times \ldots \times \mathscr{L}$. By multilinearity, we can restrict each test function $f$ in the $n$-point Schwinger function to be supported in a strip

$$
\{x, t:|t-i| \leqq 1 / 2\}
$$

$i \in Z$. Let $n_{i}$ be the number of arguments of the Schwinger function restricted to this strip; thus $\sum n_{i}=n$. With this notation, the Cauchy integral formula combined with the bound (2.9) shows that the $n$-point Schwinger function is bounded by $O\left(\prod_{i} n_{i} !\right) K^{n}$, as a functional on $\prod^{n} \mathscr{L}, K=O\left(\int\left\|\left(|x|^{2}+1\right) \cdot\right\|_{d / d-1} d t+\|\cdot\|_{d / d-1}^{d / d-1}\right)$. This bound is uniform in $n$ and in the space cutoff region. We know that the space-cutoff Wightman functions converge [15] (after selection of a subsequence), and by analytic continuation the $V \rightarrow \infty$ limit of the Schwinger functions exists also. The $V=\infty$ Schwinger functions have the same $\mathscr{L}$ norm, $O\left(\prod n_{i} ! K^{n}\right)$, which allows summation of the power series. The resulting functional $J(f)$ is complex analytic, satisfies (2.9), and is the limit of the finite volume $J$ 's. The axioms of Fröhlich are readily verified, because the estimates and positivity conditions are preserved in the $V=\infty$ limit. We only comment that his axiom CI, which yields sharp time fields, is proved using the second order estimate of [15, Lemma $1.1]$,

$$
\pm \phi(f)^{2} \leqq|f|_{\mathscr{S}}^{2}(H+I)^{2}
$$

where $|f|_{\mathscr{S}}$ is a Schwartz space norm on $f \in \mathscr{S}$, independent of $V \leqq \infty$.

From [10], we thus obtain the existence of an imaginary time field theory over $\mathscr{S}^{\prime}\left(R^{2}\right)$ and an Osterwalder-Schrader reconstructed field theory whose Schwinger functions are just the functional derivatives of $J(f)$. Thus the reconstructed Schwinger functions (and Wightman functions) agree with those of the field theory on $\mathscr{H}$, and so the two field theories are unitarily equivalent [15]. 
Theorem 2.4. Let $1 \leqq j_{v} \leqq d=\operatorname{deg} \mathscr{P}, 1 \leqq v \leqq n$. The generalized Schwinger functions

$$
\mathscr{S}_{j}^{(n)}(x)=\int \prod_{v=1}^{n}: \phi^{j_{v}}\left(x_{v}\right): d q
$$

are continuous as multilinear forms on $\prod_{v=1}^{n} \mathscr{L}_{1, d / d-j_{v}}$, with norms bounded by $\left(\prod n_{i} !\right) K^{n}$ as above. They are the functional derivatives of

$$
Z\left(h_{1}, \ldots, h_{d}\right)=\int e^{-\sum_{j=1}^{d}: \phi^{\jmath}\left(h_{j}\right):} d q .
$$

$Z$ is bounded and analytic in $h_{j} \in \mathscr{L}_{1, d / d-j}$.

Proof. The proof is the same as that of Theorem 2.3, once we know that the $V \rightarrow \infty$ limit of the generalized Schwinger functions exists. We insert a momentum cutoff $\kappa$ and use the bounds which result from Theorem 2.2. We conclude that

$$
\mathscr{L}_{j, \kappa}^{(n)}=\int \prod_{v=1}^{n}: \phi_{\kappa}^{j_{v}}(x): d q
$$

is bounded uniformly in $\kappa$ and $V$, and that

$$
\mathscr{S}_{j, \kappa}^{(n)} \rightarrow \mathscr{S}_{j}^{(n)}
$$

uniformly in $V$. Since $\mathscr{S}_{j, \kappa}^{(n)}$ can be expressed in terms of ordinary Schwinger functions by undoing the Wick order, the existence of the $V \rightarrow \infty$ limit in $\mathscr{S}_{j, \kappa}^{(n)}$ follows. By a $3 \varepsilon$ argument, the $V \rightarrow \infty$ limit exists in $\mathscr{S}_{n}^{(n)}$ also, completing the proof.

Remark. The limit (2.11) holds for $V=\infty$ also and it holds for a.e. phase $\xi \in \Xi$ uniformly in $\xi$. We next show that the estimates of Theorem 2.4 on the generalized Schwinger functions are valid in a.e. phase.

Theorem 2.5. For the $\mathscr{P}(\phi)_{2}$ field theory acting on $\mathscr{H}(\xi)$, the Schwinger functions satisfy the bounds of Theorem 2.4, uniformly in $\xi$, and are the functional derivatives of a complex analytic function $Z_{\xi}\left(h_{1}, \ldots, h_{d}\right)$, bounded as in Theorem 2.4 .

Proof. For test functions smooth in $t$, the Schwinger functions with $\phi(x)$, $: \phi_{\kappa}^{j}(x)$ : and $: \phi_{\kappa}^{j}(x):-: \phi_{\kappa^{\prime}}^{j}(x)$ : vertices are defined, using the estimate of Theorem 2.1 in the argument of [13]. For $E$ a projection in $\mathscr{Z}$, the same statement applies to the Schwinger functions $\mathscr{S}_{E}$ defined in the vacuum $\Omega_{E}=E \Omega$. Assuming that $h$ has compact support in $t$, we bound $\mathscr{S}_{E}^{(N)}$ by $K^{N}\left(\prod_{i} N_{i} !\right)\|E \Omega\|^{2}$, using the Schwarz inequality:

$$
\begin{aligned}
\left|\left\langle\Omega_{E} A \Omega_{E}\right\rangle\right| & \leqq\|E \Omega\|\left\langle\Omega_{E} A^{*} A \Omega_{E}\right\rangle^{1 / 2} \\
& \leqq \ldots \leqq\|E \Omega\|^{2^{-2^{n}}}\left\langle\Omega\left(A^{*} A\right)^{2^{n}} \Omega\right\rangle^{2^{-n-1}}
\end{aligned}
$$

Here $A$ is the integral of a time ordered product of $\phi(x), \ldots$ vertices as above and of $e^{-t H}$ operators. We suppose that the test functions for $A$ also have compact support, and we choose the strips in Theorem 2.4 to match the support of the test functions in $A$. Then $\left(A^{*} A\right)^{2^{n}}$ is also such a time ordered product, with test functions supported in $2^{n+1}$ times as many time strips. If $A$ is defined by $N$ functional derivatives of $Z,\left(A^{*} A\right)^{n}$ has $2^{n+1} N$ such functional derivatives; however, only $N_{i}$ occur in a given time strip. Thus

$$
\left\langle\Omega\left(A^{*} A\right)^{2^{n}} \Omega\right\rangle \leqq\left(K^{N} \prod_{i} N_{i} !\right)^{2 n+1}
$$


which yields

$$
\left|\left\langle\Omega_{E} A \Omega_{E}\right\rangle\right| \leqq\|E \Omega\|^{2} K^{N} \prod_{i} N_{i} ! .
$$

Thus we see that $\mathscr{S}_{E}^{(n)} /\|E \Omega\|^{2}$ satisfies the bounds of Theorem 2.4, unformly in $E$. Passing to the pure phases, we have

$$
\begin{aligned}
\mathscr{S}_{E}^{(n)} & =\int_{\Xi_{E}} \mathscr{S}_{\xi}^{(n)} d \xi \\
\|E \Omega\|^{2} & =\int_{\Xi_{E}} d \xi,
\end{aligned}
$$

which $\Xi_{E}$ is the subset of $\Xi$ corresponding to $E \in \mathscr{Z}$. It follows that $\mathscr{S}_{\xi}^{(n)}$ is $L_{\infty}(\Xi, d \xi)$, as a functional on $\mathscr{L}_{1, d / d-j_{1}} \times \ldots \times \mathscr{L}_{1, d / d-j_{n}}$ depending on $\xi$. These bounds permit summation of the exponential series, yielding $Z_{\xi}$, for a.e. $\xi$. They also permit removal of the ultraviolet cutoff in the $\phi^{j}(x)$ vertices. This completes the proof.

Results similar to Theorems 2.3-2.5 were obtained independently by Fröhlich [11]. As a reformulation of results of [11], we have the following two theorems:

Theorem 2.6. For the $\mathscr{P}(\phi)_{2}$ field theory acting on $\mathscr{H}(\xi)$, a.e. pure phase is unitarily equivalent to an Osterwalder-Schrader reconstructed theory. If $d q_{\xi}$ denotes the associated path space measure on $\mathscr{S}^{\prime}\left(R^{2}\right)$, then

$$
Z=\int Z_{\xi} d q \text {. }
$$

Theorem 2.7. Under the unitary equivalence of Theorem 2.3, $\mathscr{Z}=\mathfrak{U}^{\prime}$ is identified with the algebra $\mathscr{M}_{\mathrm{inv}}$ of time translation invariant bounded measurable function on $\mathscr{S}^{\prime}\left(R^{2}\right)$.

Proof. By [11, Lemma 2.17], $\mathscr{M}_{\text {inv }} \subset \mathscr{M}_{\infty} \cap \mathscr{M}_{+} \cap \mathscr{M}_{-}$, where $\mathscr{M}_{\infty}$ is $L_{\infty}$ of the $\sigma$-field $\sum_{\infty}$ of sets measurable at $\infty$, and $\mathscr{M}_{ \pm}$is $L_{\infty}$ of the $\sigma$-field $\sum_{ \pm}$generated by functions $\phi(x, t)$, for $\pm t \geqq 0$. Let $G$ be a positive element of $\mathscr{M}_{\text {inv }}$. Then $G$ is invariant under the time reflection operator $\theta$ [11, Proof of Theorem 2.19]. Let $E_{\mathrm{inv}}$ be the conditional expectation projection onto $\mathscr{M}_{\text {inv }}$. Then for $f \in \mathscr{M}_{+}$,

$$
\begin{aligned}
0 & \leqq \int(\theta G f)^{-} G f d q \\
& =\int(\theta f)^{-} f G^{2} d q
\end{aligned}
$$

and since this is true for all $G \geqq 0$ in $\mathscr{M}_{\text {inv }}$,

$$
0 \leqq E_{\text {inv }}(\theta f)^{-} f \text {. }
$$

Hence

$$
\begin{aligned}
\int(\theta G f)^{-} G f d q & \leqq\left\|G^{2}\right\|_{\infty} \int E_{\mathrm{inv}}(\theta f)^{-} f d q \\
& =\left\|G^{2}\right\|_{\infty} \int(\theta f)^{-} f d q=\left\|G^{2}\right\|_{\infty}\langle f, f\rangle .
\end{aligned}
$$

It follows that $G$ defines a bounded operator on $\mathscr{H}$. $G$ is seen to commute with $\mathscr{M}_{0}$, the functions of the time zero field, and $G$ commutes with time translation, on $\mathscr{H}$. Thus $G \in \mathfrak{U}^{\prime}=\mathscr{Z}$.

We have proved that $\mathscr{M}_{\text {inv }}$ is a subalgebra of $\mathscr{Z}$. Thus we can define a direct integral decomposition of $\mathfrak{U}$ with respect to $\mathscr{M}_{\text {inv }}$. Each component $\mathfrak{A}(\zeta)$ is an Osterwalder-Schrader reconstruction with path space measure $d q_{\zeta}$ equal to a component of $d q$ under direct integral decomposition of $d q$ with respect to $\mathscr{M}_{\text {inv }}$. Thus the measures $d q_{\zeta}$ are ergodic under time translation [11, Theorem 2.16]. 
Hence they have a cluster property, from which the irreducibility of $\mathfrak{U}(\zeta)$ follows. By general properties of $C^{*}$-algebra decompositions, this shows that $\mathscr{Z}=\mathscr{M}_{\text {inv }}$.

\subsection{Integration by Parts}

The Green's functions $\mathscr{G}^{(n)}$ are defined below as the connected (truncated) parts of the Schwinger functions $\mathscr{S}^{(n)}$, with the truncation defined in the standard fashion. For the case at hand, namely a direct integral of irreducible theories, the truncation is performed in each pure phase $\xi$, defining $\mathscr{G}_{\xi}^{(n)}$, and then by definition $\mathscr{G}^{(n)}=\int \mathscr{G}_{\xi}^{(n)} d \xi$. The ultraviolet behavior of $\mathscr{S}^{(n)}$ and $\mathscr{G}^{(n)}$ are determined by perturbation theory, and these singularities coincide with the local singularities of a massive free theory. In order to establish this fact, we integrate by parts on path space. Successive integration by parts yields a representation of $\mathscr{S}^{(n)}$ or $\mathscr{G}^{(n)}$ as a sum of low order perturbation contributions, plus a remainder which is regular in $x$. Such an expansion is possible because the $\mathscr{P}(\phi)_{2}$ model is superrenormalizable.

The formula for integration by parts in the $\mathscr{S}^{(n)}$ is

$$
\int: \phi^{j}:(h) R(\phi) d q=\int d q \int A(x)\left[\frac{\delta R}{\delta \phi(x)}-R(\phi) \mathscr{P}^{\prime}(\phi(x))\right] d x,
$$

where

$$
\begin{aligned}
A(x) & =\int d y C(x-y) h(y): \phi^{j-1}:(y), \\
R(\phi) & =\prod_{i=1}^{n}: \phi^{j_{i}}:\left(h_{i}\right) . \\
C & =\left(-\Delta+m_{0}^{2}\right)^{-1} .
\end{aligned}
$$

Theorem 3.1.1. The partial integration formula (3.1.1) is valid for the $V=\infty$ field theory and in a.e. phase $\xi \in \Xi$.

Remark. The left side of (3.1.1) is defined by Theorem 2.6 and 2.10, while the right side of (3.1.1) has kernels which do not factor. To deal with this situation, we integrate by parts in each linear factor in $: \phi^{j}:(h) R(\phi)$. This result produces a sum of many terms, of the form

$$
W=\int\left[\prod_{v=1}^{r}: \mathscr{P}^{\left(\alpha_{v}\right)}\left(\phi\left(y_{v}\right)\right):\right] w(y) d y,
$$

where

$$
w(y)=\int v(x, y) \prod_{i=1}^{n} h_{i}\left(x_{i}\right) d x,
$$

and where $v(x, y)$ is a constant times a product of factors $C\left(x_{i}-y_{j}\right)$ and $C\left(x_{i}-x_{j}\right)$.

Let $\mu^{-\varepsilon}$ be the operator $\left(-\Delta+m_{0}^{2}\right)^{-\varepsilon / 2}$, and let $\mu_{\otimes j}^{-\varepsilon}$ be the product of $\mu^{-\varepsilon}$ acting on $j$ variables.

Lemma 3.1.2. In (3.1.2), $\alpha_{v} \geqq 1$ and $C(x) \in \mathscr{L}_{d / \alpha_{v}}$.

Proof. By definition $\alpha_{v} \geqq 1$, so $d / \alpha_{v}<\infty$. Also $C(x)$ is $L_{p}$ for all $p<\infty$.

Lemma 3.1.3. For $\varepsilon<0$ and any choice of exponents $l_{v}$ in $Z^{+}$, the function

$$
\mu^{-\varepsilon} \prod_{v=1}^{r} C\left(x-y_{v}\right)^{l_{v}}
$$

is bounded and continuous. Here $\mu^{-\varepsilon}$ acts on $x$. 
Proof. $C\left(x-y_{v}\right)$ has a Fourier transform of magnitude $\left(p^{2}+m_{0}^{2}\right)^{-1}$. Thus the $l=\sum l_{\nu}$ fold convolution is bounded by $O(1)\left(p^{2}+m_{0}^{2}\right)^{-1+\varepsilon / 2}$, by standard power counting estimates, see e.g. [19]. After multiplication by $\mu^{-\varepsilon}$, the Fourier transform is integrable, and hence (3.1.3) is bounded. Continuity follows similarly, since $\left|\left(e^{i p x}-1\right)\right| \leqq O\left(|p x|^{\varepsilon / 3}\right)$.

Theorem 3.1.4. For any $\varepsilon>0, \mu_{\otimes}^{-\varepsilon} \mathscr{S}^{(n)}$ is a bounded continuous function, with bounds uniform in $\xi \in \Xi$.

Theorem 3.1.5. Let $\mathscr{C}_{\{j\}}=\prod_{1 \leqq i<i^{\prime} \leqq n}\left(1+C\left(x_{i}, x_{i^{\prime}}\right)^{\left(j_{i}+j_{i}\right)}\right)$. Then $\mathscr{C}_{\{j\}}^{-1} \mathscr{S}^{(n)}$ is a bounded continuous function, with bounds uniform in $\xi \in \Xi$.

Proof of Theorems 3.1.1, 3.1.4, 3.1.5. The integration by parts formula (3.1.1) is known for $V<\infty$. In that case by Theorem 2.6, the representation (3.1.2) and the Lemmas 3.1.2, 3.1.3 above,

$$
\begin{aligned}
\left|\left(\mu_{\otimes n}^{-\varepsilon} \mathscr{S}^{(n)}\right)(h)\right| & =\left|\mathscr{S}^{(n)}\left(\mu_{\otimes n}^{-\varepsilon} h\right)\right| \\
& \leqq \sum_{\text {finite sum }} \int d x \prod\left\|C\left(y_{v}-x_{i}\right)\right\|_{\mathscr{L}_{a / \alpha_{v}}} \prod C\left(x_{i}-x_{j}\right)\left|\mu_{\otimes n}^{-\varepsilon} h\right| \\
& \leqq \sum_{\text {finite sum }} O(1) \int d x \prod C\left(x_{i}-x_{j}\right) \mu_{\otimes n}^{-\varepsilon}|h| \\
& \leqq \sum_{\text {finite sum }} O(1) \int|h| \mu_{\otimes n}^{-\varepsilon} \prod C\left(x_{i}-x_{j}\right) \\
& \leqq O(1)\|h\|_{\mathscr{L}_{1}},
\end{aligned}
$$

where $h=\prod_{i=1}^{n} h_{i}\left(x_{i}\right)$. Since the dual of $\mathscr{L}^{1}$ is $\mathscr{L}^{\infty}$, in a finite volume $\mu_{\otimes n^{-\varepsilon} \mathscr{Y}^{(n)}}$ is $\mathscr{L}^{\infty}$. Since $\mu_{\otimes n}^{-\varepsilon}$ maps $\mathscr{L}^{\infty}$ into bounded, continuous functions, Theorem 3.1.4 holds for $V<\infty$, with bounds uniform in $V$. Similarly, Theorem 3.1 .5 holds for $V<\infty$ with bounds uniform in $V$. By limits, the same bounds are valid for $V=\infty$. As in Chapter 2, these estimates decompose into a.e. phase $\xi$. This establishes the existence of the right side of (3.1.1). The existence of the $V=\infty$ limit for (3.1.1) follows as above.

For a polynomial $Q(\phi)$, let $Q_{T}=T^{-1} \int_{0}^{T} Q(\phi(\cdot+t)) d t$. Then $Q_{T}$ is bounded in $L_{p}$, for all $T<\infty$ and $p<\infty$. Let $Q_{\infty}$ be any $w^{*}-L_{p}$ limit point. In the integration by parts formula, if we insert a factor $Q_{T}$ in both sides, a new term is included due to contraction to $Q_{T}$. This term vanishes, however, as $T \rightarrow \infty$. Thus the formula remains valid even when the integrands on both sides are multiplied by $Q_{\infty}$.

Now $Q_{\infty}$ is time translation invariant, and thus bounded functions of $Q_{\infty}$ belong to $\mathscr{Z}$, the center of $\mathfrak{U}$. Let $\mathscr{Z}_{\infty}$ denote the subalgebra of $\mathscr{Z}$, generated by all such $Q_{\infty}$. Then integration by parts remains valid in the direct integral theory, decomposed with respect to $\mathscr{Z}_{\infty}$. We must only show that a theory for which $\mathscr{Z}_{\infty}=\{$ constants $\}$ is irreducible.

We show reducibility implies $\mathscr{Z} \neq\{$ constants $\}$. Consider a theory with a multiple vacuum and let $P$ denote the projection onto the space of vacuum vectors. Suppose $R$ is a polynomial such that $R \Omega \perp \Omega, P \Omega=\Omega$, and $\|P R \Omega\|=1$. Then

$$
1=\lim _{T \rightarrow \infty} \frac{1}{T} \int_{0}^{T} d t\left\langle R \Omega, e^{-t H} R \Omega\right\rangle=\left\langle R \Omega, R_{\infty} \Omega\right\rangle
$$


and

$$
0=\left\langle\Omega, e^{-t H} R \Omega\right\rangle=\left\langle\Omega, R_{\infty} \Omega\right\rangle .
$$

Thus const. $\neq R_{\infty} \in \mathscr{Z}_{\infty}$, and the proof is complete.

\subsection{Green's Functions and Graphs}

The integration by parts formula will be used in our study of Green's functions, i.e. connected or truncated Schwinger functions. Here we define truncations for the generalized Green's functions and relate these to the integration by parts formula.

Let $A_{1}, \ldots, A_{n}$ be functions of $\phi$, e.g. $A_{i}=: \phi^{j_{r}}:\left(h_{i}\right)$. Let $P$ be the set of partitions of $\{1, \ldots, n\}$ and for $\varrho \in P$, let $\varrho=\left\{\sigma_{1}, \ldots, \sigma_{j}\right\},|\varrho|=j$. Each $\sigma_{v}$ is a subset of $\{1, \ldots, n\}$.

Definition 3.2.1. $\left\langle\prod_{i} A_{i}\right\rangle_{T}$ is defined recursively by

$$
\left\langle A_{1} \ldots A_{n}\right\rangle=\sum_{\varrho \in P} \prod_{\sigma \in \varrho}\left\langle\prod_{i \in \sigma} A_{i}\right\rangle_{T} .
$$

Remark. $\left\langle\prod A_{i}\right\rangle_{T}$ depends not only on $\prod A_{i}$, but also on its factorization into the $A_{i}$ 's.

Proposition 3.2.2. The inversion of (3.2.1) is

$$
\left\langle A_{1} \ldots A_{n}\right\rangle_{T}=\sum_{\varrho \in P}(-1)^{|\varrho|}(|\varrho|-1) ! \prod_{\sigma \in \varrho}\left\langle\prod_{i \in \sigma} A_{i}\right\rangle \text {. }
$$

Proof. This is standard.

Consider the case that each $A_{i}$ is a monomial in the field. Successive integration by parts produces a sum of terms, and each term is labelled by a graph $D$. If $\mathscr{D}$ is the set of all such graphs, the identity has the form

$$
\left\langle\prod_{i} A_{i}\right\rangle=\sum_{D \in \mathscr{D}}\left\langle\prod_{\nu} B_{v}\right\rangle
$$

where $B_{v}$ are monomials in the field [formed from derivatives of the $A_{i}$ or of $\mathscr{P}$, as in (3.1.1)] and determined by $D$. The vertices of the graph are the $B_{v}$, while the lines arise from contractions $C\left(x-x^{\prime}\right)$.

Theorem 3.2.2. With the above definitions of $\mathscr{D}$ and $B_{v}$

$$
\left\langle\prod_{i=1}^{n} A_{i}\right\rangle_{T}=\sum_{D \in \mathscr{D}}\left\langle\prod_{v} B_{v}\right\rangle_{T} .
$$

Proof. It is sufficient to treat the case of one integration by parts, in one linear factor from $A_{1}$. We use (3.2.2) to define $\left\langle\prod A_{i}\right\rangle_{T}$, and integrate by parts on the right side in the factor containing $A_{1}$. We group the result into $n+1$ terms. There are $n-1$ terms in which $A_{1}$ contracts to an $A_{i}, 1 \leqq i \leqq n$. There is one term with $A_{1}$ contracted to a new : $\mathscr{P}(\phi)$ : vertex, and if $A_{1}$ is not Wick ordered, a contraction of $A_{1}$ to itself is also possible. Let $B_{1}^{\prime}$ be the function of $\phi$ which results from the contraction of $A_{1}$ to : $\mathscr{P}$ :, and let $B_{1}^{\prime \prime}$ be the result of contracting $A_{1}$ to itself. Since these last two terms occur for each $\varrho \in P$, they contribute

$$
\begin{gathered}
\left.\sum_{\varrho \in P}(-1)^{|\varrho|}(|\varrho|-1) !\left(\prod_{\substack{\sigma \in \varrho \\
1 \notin \sigma}}\left\langle\prod_{i \in \sigma} A_{i}\right\rangle\right)\left\langle\left(\prod_{\substack{i \in \sigma_{i} \\
i \neq 1}} A_{i}\right) B_{1}^{\prime}+B_{1}^{\prime \prime}\right)\right\rangle \\
=\left\langle B_{1}^{\prime} \prod_{i>1} A_{i}\right\rangle_{T}+\left\langle B_{1}^{\prime \prime} \prod_{i>1} A_{i}\right\rangle_{T}
\end{gathered}
$$


Next consider a term with $A_{1}$ contracted to $A_{i}$, and let $B_{1 i}$ be the result of this contraction. Such a term arises only for certain $\varrho \in P$, namely $\varrho$ which do not separate 1 and $i$. Let $P_{i}$ be the set of all such partitions. This term is

$$
\begin{gathered}
\sum_{\varrho \in P_{\mathfrak{t}}}(-1)^{|\varrho|}(|\varrho|-1) !\left(\prod_{\substack{\sigma \in \varrho \\
1 \neq \sigma}}\left\langle\prod_{i \in \sigma} A_{i}\right\rangle\right)\left\langle B_{1 i} \prod_{\substack{v \in \sigma_{1} \\
1 \neq v \neq i}} A_{v}\right\rangle \\
=\left\langle B_{1 i} \prod_{1 \neq v \neq i} A_{v}\right\rangle_{T} .
\end{gathered}
$$

Combining these $n+1$ terms completes the proof of Theorem 3.2.2.

Remark 3.2.3. For any graph $D$ which is not connected, but which has a vacuum component (so that $\operatorname{deg} B_{v}=0$, or $B_{v}=$ const. for some $v$ ), the term $\left\langle\prod_{v} B_{v}\right\rangle_{T}$ vanishes.

The truncations defined by (3.2.2) are not linear in the state \langle\rangle . Thus there is a difference between truncating in the state defined by $\Omega \in \mathscr{H}$ and truncating in a.e. pure phase $\xi$. The latter is the correct definition, because it leads to Green's functions with nice cluster properties. Using the measure $d q_{\xi}$, we define the general Green's functions as

$$
G_{\xi}^{(n)}\left(x_{1}, \ldots, x_{n}\right)=\left(\int: \phi\left(x_{1}\right)^{j_{1}} \ldots . .: \phi\left(x_{n}\right)^{j_{n}: d q_{\xi}}\right)_{T}
$$

and

$$
G^{(n)}\left(x_{1}, \ldots, x_{n}\right)=\int_{\Xi} G_{\xi}^{(n)}\left(x_{1}, \ldots, x_{n}\right) d \xi .
$$

It follows immediately that $G^{(n)}$ and $G_{\xi}^{(n)}$ satisfy the bounds of Theorems 3.1.4 and 3.1.5. We improve these bounds in Chapter 4. Using integration by parts, as in Theorem 3.1.1, we no longer need to restrict $j_{v}$ to be less than $d=\operatorname{deg} \mathscr{P}$. However, in this case, we lose the nice $n$ ! bound on the $\mathscr{S}^{(n)}$ from Chapter 2 . In principle, the $G^{(n)}$ should have an $n$ ! estimate (as derivatives of an analytic function) for factorizing test functions, and for $j_{v} \leqq d$. See $[20,11]$.

We call ordinary the Green's functions

$$
G_{\xi}^{(n)}\left(x_{1}, \ldots, x_{n}\right)=\left(\int \phi\left(x_{1}\right) \ldots \phi\left(x_{n}\right) d q_{\xi}\right)_{T},
$$

or their integral, and generalized the Green's functions (3.2.4).

\section{Regularity of the Green's Functions}

There are two aspects of the regularity of the Green's functions: their short distance singularities (ultraviolet behavior) and their long distance decay (infrared behavior). The intermediate range behavior is settled by the general fact that the $\mathscr{S}^{(n)}$ are real analytic at noncoinciding points. The infrared behavior is controlled by the assumption that we are dealing with a noncritical theory. In the case of a physical mass $m>0$, the $G^{(n)}$ decay exponentially under large separation of points, $\left|G^{(n)}\right| \leqq e^{-m r}$. We assume that decay occurs in all Euclidean directions, as is the case for a Lorentz invariant theory with $m>0$. The following theorem bounds the ultraviolet behavior:

Theorem 4.1. For $n>2$, the ordinary $\mathscr{P}(\phi)_{2}$ Green's functions (3.2.6) are bounded and continuous for a.e. $\xi \in \Xi$, with bounds uniform in $\xi$. For $n=2$, 
$G_{\xi}^{(2)}\left(x-x^{\prime}\right)-C\left(x-x^{\prime}\right)$ has this property. For the generalized Green's functions of a $\mathscr{P}(\phi)_{2}$ theory, $\mu_{\otimes n}^{-\varepsilon} G_{\xi}^{(n)}$ and $\mathscr{C}_{\{j\}}^{-1} G_{\xi}^{(n)}$ are bounded and continuous for a.e. $\xi \in \Xi$ (cf. Theorems 3.1.4-3.1.5), with bounds uniform $\xi$.

Proof. The bounds on $\mu_{\otimes n}^{-\varepsilon} G^{(n)}$ and $\mathscr{C}_{\{j\}}^{-1} G^{(n)}$ follow from Theorems 3.1.4-3.1.5 and the definition of $G^{(n)}$. The bound on the ordinary Green's functions (all $j_{v}=1$ ) follows from the proof of these theorems, once we observe that there are no $\phi\left(x_{i}\right)-\phi\left(x_{j}\right)$ contractions in $G^{(n)}$, because of the truncations. Here we require $n>2$, or if $n=2$, we exclude the one (zero order) term with such a contraction.

For the long distance behavior we use the comparison function

$$
\mathscr{E}_{\varepsilon}=\prod_{1 \leqq i<l \leqq n} e^{\varepsilon\left|x_{t}-x_{l}\right|}
$$

Theorem 4.2. For a noncritical $\mathscr{P}(\phi)_{2}$ model with exponential decay $\geqq m$ in all Euclidean directions, the functions

$$
\mathscr{E}_{\varepsilon} \mu_{\otimes n}^{-\delta} G^{(n)}, \quad \mathscr{E}_{\varepsilon} \mathscr{C}_{\{j\}}^{-1} G^{(n)}
$$

are bounded and continuous for some $\varepsilon>0$ and for any $\delta>0$. For the ordinary Green's functions, the same is true for

$$
\mathscr{E}_{\varepsilon} G^{(n)} \text {. }
$$

Proof. We modify the proof of Theorems 3.1.4-3.1.5 to obtain the exponential decay. Let $d$ be the diameter of $\left\{x_{1}, \ldots, x_{n}\right\}$, and let $\left(x_{i}, x_{j}\right)$ be the pair determining this diameter. We consider two parallel lines separated by distance $r$ which are perpendicular to the line $\left(x_{i}, x_{j}\right)$ and which separate $\left\{x_{1}, \ldots, x_{n}\right\}$ into two nonempty clusters $\left\{I_{1}, I_{2}\right\}$. We assume each $x_{i}$ has a distance at least $r$ from the lines. We assert such lines always exist with $r \geqq d / 3 n$, see [30].

For a term labelled by a graph $D$, let $\varrho(D)$ be the partition of $\{1, \ldots, n\}$ determined by the connected components of $D$. If $\varrho$ does not refine $\left\{I_{1}, I_{2}\right\}$, then $G$ contains a sequence of contractions of length $r$. In this case, the term is dominated by $O\left(e^{-m_{0} r}\right)$, so the corresponding contributions to $G^{(n)}$ have exponential decay. Thus we consider partitions $\varrho$ which refine $\left\{I_{1}, I_{2}\right\}$. In each integration $d z$ over a generalized Green's function in a connected component, we restrict $d z$ to lie on the same side of the parallel lines as the $x_{i}$ variables of that component. The contribution from the complementary $z$ region is again exponentially small. However, the contribution with $z$ restricted in this fashion is also exponentially small, as one sees by using the Schwarz inequality and the mass $m>0$ in a standard fashion.

We now obtain expressions for the constant momentum subspace of $H_{-1}^{\otimes j}$ and for the restriction of $G^{(n)}$ to this subspace. In $L_{2}\left(R^{2 j}\right)=L_{2}\left(R^{2}\right)^{\otimes j}$, the functions $f\left(x_{1}, \ldots, x_{n}\right)$ of zero total momentum are invariant under the transformation $x_{i} \rightarrow x_{i}+a$. We define a norm on such functions

$$
|f|=\left[\int\left|f\left(x_{1}, \ldots, x_{n}\right)\right|^{2} d x_{2} \ldots d x_{n}\right]^{1 / 2} .
$$

namely the $L_{2}$ norm in $x_{2}, \ldots, x_{n}$, and we let $\mathscr{H}(0)$ denote the zero momentum Hilbert space. Functions of total momentum $P$ have the form

$$
f_{P}=e^{i P \sum x_{j} / n} f \in \mathscr{H}(P), \quad f \in \mathscr{H}(0),
$$

and $\left|f_{P}\right|=|f|$. We also write $\mathscr{H}^{(n)}(P)$ to indicate the number of particles. 
The norm in $H_{ \pm 1}^{\otimes j}$ is determined by $\mu_{\otimes j}^{ \pm 1}$ and the mappings

$$
H_{1}^{\otimes j} \underset{\mu \otimes_{j}}{\longrightarrow} L_{2}\left(R^{2}\right)^{\otimes j} \underset{\mu \otimes_{J}}{\longrightarrow} H_{-1}^{\otimes j}
$$

are unitary. We define the momentum $P$ vectors $\mathscr{H}_{ \pm}(P)$ in $H_{-1}^{\otimes j}$ by

$$
\mathscr{H}_{ \pm}(P)=\mu_{\otimes}^{ \pm 1} \mathscr{H}(P),
$$

with the norm $\left|\mu_{\otimes j}^{ \pm 1} f_{P}\right|=\left|f_{P}\right|_{ \pm}$.

We consider $G^{(n)}\left(x_{1}, \ldots, x_{n}\right)$ as the kernel of an integral operator $G^{(n)}$ from $H_{-1}^{\otimes j}$ to $H_{1}^{\otimes n-j}$. This is unitarily equivalent to the operator

$$
\mu_{\otimes n} G^{(n)} \equiv \mu_{\otimes n-j} G^{(n)} \mu_{\otimes j}
$$

as a transformation from $L_{2}^{\otimes j}$ to $L_{2}^{\otimes n-j}$. The unitary equivalence commutes with translations.

If we extend $G^{(n)}$ to a map from $\mathscr{H}_{-}^{(j)}(P)$ to $\mathscr{H}_{+}^{(n n-j)}(P)$, this is unitarily equivalent to $\mu_{\otimes n} G^{(n)}$ as a map from $\mathscr{H}^{(j)}(P)$ to $\mathscr{H}^{(n-j)}(P)$.

Theorem 4.3. (a) For a noncritical $\mathscr{P}(\phi)_{2}$ model, and $0<j<n$, the ordinary Green's functions $G^{(n)}$ is a bounded operator from $H_{-1}^{\otimes j}$ to $H_{1}^{\otimes n-j}$.

(b) If in addition $n>2$, then $G^{(n)}$ defines a Hilbert-Schmidt map, $\mu_{\otimes n} G^{(n)}$, from $\mathscr{H}^{(j)}(P)$ to $\mathscr{H}^{(n-j)}(P)$.

Remark. In essence, this theorem states that after differentiating each variable of $G^{(n)}$ once, the result $\mu_{\otimes n} G^{(n)}$ is an $L_{2}$ function of the difference variables. The proof also shows that $\mu_{\otimes n} G^{(n)}$ is continuous in $P$, and vanishes in Hilbert-Schmidt norm as $P \rightarrow \infty$.

Theorem 4.4. For a noncritical $\mathscr{P}(\phi)_{2}$ model, and $0<j<n$, the generalized $G^{(n)}$ is a bounded operator from $H_{-1+\varepsilon}^{\otimes j}$ to $H_{1-\varepsilon}^{\otimes n-j}$, for $\varepsilon>0$.

We prove Theorem 4.3; the proof of Theorem 4.4 is similar. We integrate by parts in each linear factor $\phi\left(x_{i}\right)$ in $G^{(n)}$. Because of truncation (see Theorem 3.2.2) there are no $\phi\left(x_{i}\right)-\phi\left(x_{k}\right)$ contractions. If $n \leqq \operatorname{deg} \mathscr{P}$, there is one factor proportional to

$$
\int \prod_{i=1}^{n} C\left(x_{i}-z\right) d z=F\left(x_{1}, \ldots, x_{n}\right)
$$

and this term fulfills the conclusions of the theorem. In fact, the Fourier transform of $\mu_{\otimes n} F$ is proportional to

$$
\delta\left(\sum_{i=1}^{n} p_{i}\right) \prod_{i=1}^{n} \mu\left(p_{i}\right)^{-1},
$$

which is the kernel of a bounded operator from $L_{2}^{\otimes_{j}}$ to $L_{2}^{\otimes_{n}-j}$, verifying the conclusion of (a). Furthermore,

$$
\begin{aligned}
\left|\mu_{\otimes n} F\right|^{2} & =\text { const. }\left\|\mu\left(\sum_{i=2}^{n} p_{i}\right)^{-1} \prod_{i=2}^{n} \mu\left(p_{i}\right)\right\|_{L_{2}}^{2} \\
& \leqq \text { const. }
\end{aligned}
$$

proving that (4.2) also satisfies the conclusion of (b).

The remaining terms are all of the form

$$
T=\int \prod C\left(x_{i}-y_{l_{\imath}}\right) G^{(r)}\left(y_{1}, \ldots, y_{r}\right) d y,
$$


where $G^{(r)}$ is a generalized Green's function. By Theorem $4.2, \mu_{\otimes n} T$ is continuous, and exponentially decreasing in its difference variables. The theorem follows from these facts.

\section{The Support of $d q$}

Following Fröhlich [11], we introduce the Euclidean $\pi$ operators which generate the translation group $\phi \rightarrow \phi+f$.

$$
e^{i \pi(f)}: F(\phi) \rightarrow F(\phi+f)\left(\frac{d(q+f)}{d q}\right)^{1 / 2} .
$$

The Jacobian in (5.1) is

$$
\frac{d(q+f)}{d q}=\exp -\left[\delta \mathscr{P}(f)+\phi\left(\left(-\Delta+m_{0}^{2}\right) f\right)+\frac{1}{2}\left\langle f,\left(-\Delta+m_{0}^{2}\right) f\right\rangle\right],
$$

where

$$
\delta \mathscr{P}(f)=\int[: \mathscr{P}(\phi+f):-: \mathscr{P}(\phi):] d x .
$$

In view of the bounds of Chapter 2, we choose

$$
f \in \mathscr{L}_{1, d} \cap\left(-\Delta+m_{0}^{2}\right)^{-1} \mathscr{L}_{1, d / d-1} \cap H_{1},
$$

where $H_{1}$ is a Sobolev space. Then (5.2) belongs to $L_{p}\left(\mathscr{S}^{\prime}\left(R^{2}\right), d q\right)$ for all $p<\infty$.

Theorem 5.1 [11]. The Euclidean $\pi$ operators are self adjoint operators on the Euclidean space $\mathscr{H}_{\mathscr{E}}=L_{2}\left(\mathscr{S}^{\prime}\left(R^{2}\right), d q\right)$. The generating functional

$$
E(f, g)=\left\langle\Omega, e^{i \phi(g)} e^{i \pi(f)} \Omega\right\rangle
$$

is entire analytic for $g \in L_{1, d / d-1}$ and $f$ in (5.3).

Theorem 5.2. The Euclidean operators exist as self adjoint operators on $\mathscr{H}_{\delta}(\xi)=L_{2}\left(\mathscr{S}^{\prime}\left(R^{2}\right), d q_{\xi}\right)$. The generating functional $E_{\xi}$, defined as in (5.4), characterizes the Weyl form of the commutation relations. (It satisfies positive definiteness, continuity and commutation relations.) Moreover $E=\int E_{\xi} d \xi$, and $E_{\xi}$ satisfies the bounds of Theorem 5.1.

This theorem is essentially contained in [11]. The key step is to show that (5.2) is the Jacobean of the translated measure on $\mathscr{H}_{\mathscr{E}}(\xi)$.

It is convenient to work with a domain of smooth vectors $\mathscr{D}$ which is dense in $\mathscr{H}_{\mathscr{E}}(\xi)$. Let $\mathscr{D}$ be the linear span of $\Omega \equiv 1$ and the vectors

$$
A \Omega, \quad A=A_{n}=\left(\prod_{i=1}^{n} \phi\right)\left(g_{n}\right),
$$

where $g_{n}$ is a symmetric function in $\mathscr{S}\left(R^{2}\right)^{\otimes n}$. Then

$$
\begin{aligned}
& \phi(f) \mathscr{D} \subset \mathscr{D}, \quad f \in \mathscr{S}\left(R^{2}\right), \\
& U(a) \mathscr{D} \subset \mathscr{D},
\end{aligned}
$$

where we assume that $\Omega$ is invariant under the action of Euclidean translations $U(a) . \mathscr{D}$ is not invariant under $\pi(f)$, and so we introduce the domain $\mathscr{D}^{\prime}$, spanned 
by the vector $\Omega$ and

$$
A \Omega, A=\prod_{i=1}^{n}: \phi^{v_{1}}\left(g_{n}\right):, \quad v_{i} \leqq d .
$$

Then

$$
: \phi^{v}(f): \mathscr{D}^{\prime} \subset \mathscr{D}^{\prime} ; \quad U(a) \mathscr{D}^{\prime} \subset \mathscr{D}^{\prime} ;
$$

for $v \leqq d$, and

$$
\pi(f) \mathscr{D}^{\prime} \subset \mathscr{D}^{\prime}, f \in \mathscr{S}\left(R^{2}\right) .
$$

The relation (5.7) follows from the canonical commutation relations and the identity

$$
\pi(f) \Omega=-\frac{i}{2}\left\{\mathscr{P}^{\prime}(f)+\phi\left(\left(-\Delta+m_{0}^{2}\right) f\right)\right\} \Omega,
$$

which is the infinitesimal form of (5.2).

Theorem 5.3. The $L_{2}$ inner product defined by $d q_{\xi}$ is nondegenerate on $\mathscr{D} \times \mathscr{D}$.

Remark. The equation $B=0$, where

$$
B=\sum_{n=0}^{N} A_{n}(q)
$$

defines a polynomial variety in $\mathscr{S}^{\prime}\left(R^{2}\right)$. The theorem states that $d q_{\xi}$ is not supported on any polynomial variety. Here $A_{n}$ is defined by (5.5).

Proof. Assume that for all $C \in \mathscr{D}, \int C B d q_{\xi}=0$. Since $B \in L_{2}\left(d q_{\xi}\right)$ and since $\mathscr{D}$ is dense in $\mathscr{D}^{\prime}$ in the $L_{2}\left(d q_{\xi}\right)$ norm, by the remark following Theorem $2.4, B \perp \mathscr{D}^{\prime}$ also. Thus

$$
0=\int C_{1} B C_{2} d q_{\xi}=\left\langle C_{1} B C_{2}\right\rangle, \quad C_{i} \in \mathscr{D}^{\prime},
$$

and so for all $f_{j} \in \mathscr{S}\left(R^{2}\right)$, we have

$$
\begin{aligned}
0 & =\left\langle\Omega\left[\pi\left(f_{1}\right),\left[\pi\left(f_{2}\right),\left[\ldots,\left[\pi\left(f_{N}\right), B\right] \ldots\right]\right]\right] \Omega\right\rangle \\
& =N !(-i)^{N} g_{N}\left(f_{1} \otimes \ldots \otimes f_{N}\right) .
\end{aligned}
$$

It follows that $g_{N} \equiv 0$, and by induction $g_{n} \equiv 0$ for all $n$. This completes the proof.

We need to improve this result by allowing $A_{n}$ to lie in a constant momentum subspace. We denote the pure phase vacuum $\int \cdot d q_{\xi}$ by $\langle\cdot\rangle=\langle\cdot\rangle_{\xi}$, as above, and we assume a nonzero mass gap.

Theorem 5.4. Assume an exponential decay rate for correlations, uniformly in all Euclidean directions. Given $p \in R^{2}$, suppose

$$
0=\int\left[\left\langle C, U(a) e^{i p a} B\right\rangle-\langle C\rangle\left\langle e^{i p a} B\right\rangle\right] d a
$$

for all $C \in \mathscr{D}$. Then

$$
0=\int g_{n}\left(x_{1}-a, \ldots, x_{n}-a\right) e^{i p a} d a, \quad n \geqq 1 .
$$

Proof. The integrand in (5.11) is rapidly decreasing in $a$, as a consequence of the nonzero mass gap and Theorem 4.2. In fact, the expansion into connected Green's functions yields a sum of products, and each product contains at least 
one factor with variables from both $B$ and $C$. This factor decays rapidly in $|a|$ by Theorem 4.2 and the fact that $g_{n} \in \mathscr{S}$, while the other factors are bounded. We then follow the proof of Theorem 5.3.

For application in Chapters 7,8, we want to consider kernels $g_{n} \in H_{-1}^{\otimes n}$ rather than in $\mathscr{S}$. In this case, $A_{n}$ given by (5.5) is not in general defined (even for the free field) but requires some subtraction, e.g. Wick ordering would be sufficient. We define the Wick ordering with respect to the measure $d q_{\xi}$, i.e. with respect to the function $\Omega \equiv 1$.

In order to analyze higher portions of the energy spectrum, it is also necessary to subtract states associated with the span of $n=1,2, \ldots$ fields, $\phi(f), \phi(f) \phi(g)$, etc. We consider here the cases $n=0,1$. We discuss briefly the $n=2$ subtraction in Chapter 8 , when we introduce equations for three particle states.

Let $P_{j}$ be the projection of $\mathscr{H}_{\mathscr{E}}(\xi)$ onto the closed subspace of degree $j$ polynomials in $\phi$ which are orthogonal to all degree $l$ polynomials, $l<j$.

Theorem 5.5. With the hypotheses of Theorem 5.4,

$$
\begin{aligned}
\left(1-P_{0}\right) B & =B-\langle B\rangle \\
P_{1} B & =-\int\langle B \hat{\phi}(y)\rangle \Gamma^{(2)}(y-z) \hat{\phi}(z) d y d z
\end{aligned}
$$

where $\hat{\phi}=\phi-\langle\phi\rangle, B$ is defined by (5.9) and each $g_{n} \in \mathscr{S}\left(R^{2 n}\right)$.

Proof. Since (5.13) is obvious, we need only verify (5.14). By Theorem 4.2, $g(y)=\langle B \hat{\phi}(y)\rangle$ is a rapidly decreasing element of $H_{1}$. Since $\Gamma^{(2)}: H_{1} \rightarrow H_{-1}$ [20], $h=\Gamma^{(2)} g \in H_{-1}$, and

$$
\langle\hat{\phi}(\bar{h}) \hat{\phi}(h)\rangle=G^{(2)}(\bar{h} \otimes h) \leqq O(1)\|h\|_{-1}^{2},
$$

cf. [20]. Thus the right side of (5.14) is in $L_{2}\left(\mathscr{S}^{\prime}\left(R^{2}\right), d q_{\xi}\right)$. Finally we note that the right side of (5.14) equals: 0 for $B=1 ; \hat{\phi}(x)$ for $B=\hat{\phi}(x)$; and 0 if $B \perp$ to 1 and $B \perp \phi(x)$, all $x$. These properties characterize $P_{1}$, hence (5.14).

Corollary 5.6. Let

$$
B=\int \hat{\phi}(x) \hat{\phi}(y) g(x, y) d x d y \text {. }
$$

Then (5.13) and (5.14) admit extensions to $g \in H_{-1}^{\otimes 2}$, by continuity.

Proof. We note

$$
\langle B \hat{\phi}(z)\rangle=\int G^{(3)}(x, y, z) g(x, y) d x d y .
$$

Thus by Theorem 4.3 , we see that (5.14) extends by continuity to $g \in H_{-1}^{\otimes 2}$. To establish (5.13) on $H_{-1}^{\otimes 2}$, we note that

$$
\left\|\left(1-P_{0}\right) B\right\|^{2}=G^{(4)}\left(g^{-} \otimes g\right)+\left\langle g, G^{(2)} \otimes G^{(2)} g\right\rangle_{L_{2}\left(R^{2}\right)},
$$

and each term extends to $H_{-1}^{\otimes 2}$ by continuity, using Theorem 4.3 and (5.14a).

We now assume the decay properties of $\Gamma^{(2)}(x)$ established in Chapter 6 , to sharpen our estimates.

Theorem 5.7. Suppose $g$ is symmetric and is exponentially decreasing in the variable $x+y$, so that for all $\beta$, with $|\beta|$ sufficiently small,

$$
e^{\beta \cdot(x+y)} g(x, y) \in H_{-1}^{\otimes 2} .
$$


Then with $B$ given by (5.15),

$$
\Gamma^{(2)} *\langle B \hat{\phi}(\cdot)\rangle \in H_{-1}
$$

is exponentially decreasing.

Proof. As above, $\langle B \hat{\phi}(\cdot)\rangle$ is exponentially decreasing, and hence an element of $H_{1, m}$, as defined in (6.9). By Theorem 6.4, and $\bar{m} \geqq m,(5.16)$ is an element of $H_{-1, m}$.

We remark that in the hypothesis of Theorem 5.4, we could write (5.11) as $0=\int\left\langle C,\left(1-P_{0}\right) U(a) e^{i p a} B\right\rangle d a$.

Theorem 5.8. With $B, g$ as above, suppose that

$$
0=\int\left\langle C,\left(1-P_{0}-P_{1}\right) U(a) e^{i p a} B\right\rangle d a
$$

for all $C \in \mathscr{D}$. Then

$$
0=\int g\left(x_{1}-a, x_{2}-a\right) e^{i p a} d a .
$$

Proof. We treat $\left(1-P_{0}\right)$ and $P_{1}$ separately, since each of these projections has rapid decay in $a$. We see this by expanding each term as a sum of products of connected Green's functions. Using the exponential decay of $g$ in the variable $x+y$, the (mass gap) exponential decay of the connected Green's functions and Theorem 6.4, the decay in a follows. Two commutators with $\pi$ then yield (5.18).

Remark 5.9. The decay of the integrand does not depend on the local regularity of $C \in \mathscr{D}$. In particular, we may take $C=B$.

\section{Decay of $\Gamma^{(2)}(x)$ and CDD Zeros}

We consider an Osterwalder-Schrader theory with the (connected) momentum space two point function

$$
\chi(p)=G^{(2)}(P)=\frac{Z}{p^{2}+m^{2}}+\int_{a \geqq M^{2}} \frac{d v(a)}{p^{2}+a} .
$$

We assume $0<m \leqq M$. If, in addition, $m<M$ and $Z \neq 0$, the model has an isolated one particle state of mass $m$, and in that case $Z$ is the field strength renormalization constant. By positivity, $Z \geqq 0$ and $d v \geqq 0$, and we assume $\int d v(a) a^{-1}<\infty$. If the theory is canonical, then in addition

$$
Z+\int d v(a)=1 \text {. }
$$

The inverse propagator $-\chi(p)^{-1}$ is also the one particle irreducible part of $\chi(p)$ The Fourier transform of $-\chi(p)^{-1}$ is $\Gamma^{(2)}(x)$, and convolution by $\Gamma^{(2)}$ is an isomorphism of $H_{1}$ onto $H_{-1}$, by [20, Theorem 2.1 and Remark 2].

We are concerned here with the exponential decay rate $\bar{m}$ of $\Gamma^{(2)}(x)$, since such decay is related to the spectral properties of the theory. We find that if $m<M$, then

$$
m<\bar{m} \leqq M,
$$

and $\bar{m}$ is related to the complex zeros of $\chi(p)$. In case $\bar{m}<M$, then the decay rate $\bar{m}$ is not associated with any physical particle. For instance, if $M$ is the mass of an 
isolated bound state, we find

$$
m<\bar{m}<M,
$$

In case the strict inequality (6.4) holds, the propagator $\chi(p)$ has a CDD zero, see Castillejo, Dalitz, and Dyson [4].

Our results are nontrivial only if $m<M$, i.e. if an upper mass gap exists in the propagator.

Definition 6.1. We define the CDD root $\bar{m}$ as the root, in the interval $(m, M]$, of the equation

$$
Z+\int_{a \geqq M^{2}} \frac{m^{2}-\bar{m}^{2}}{a-\bar{m}^{2}} d v(a)=0 .
$$

If no such root exists, or if $m=M$, define $\bar{m}=M$.

Remark 6.2. Assume a canonical theory (6.4). Then for $Z \neq 0$

$$
\bar{m}=\left[\frac{\zeta}{\zeta+Z} m^{2}+\frac{Z}{\zeta+Z} M^{2}\right]^{1 / 2},
$$

where $\zeta \in[0,1-Z]$ is defined by the equation

$$
\zeta=\int \frac{M^{2}-\bar{m}^{2}}{a-\bar{m}^{2}} d v(a)
$$

For example, if $d v(a)$ is a point mass at $a=M^{2}$, then $\bar{m}=\left[(1-Z) m^{2}+Z M^{2}\right]^{1 / 2}$, with $\zeta=1-Z$ and (6.4) holds. CDD zeros are absent if and only if

$$
\int \frac{M^{2}-m^{2}}{a-M^{2}} d v(a)<Z \text {. }
$$

We show below that $\Gamma^{(2)}(x)$ has the decay rate $\bar{m}$. In order to make precise the notion of decay rate we introduce the Sobolev-Paley-Wiener space $H_{p, \lambda}$. For $\lambda=0$, we let $H_{p, \lambda}=H_{p}$, the usual Sobolev space. For $\lambda>0$, we have

Definition 6.3. Let $H_{p, \lambda}$ be those distributions $f$ for which

$$
e^{\alpha x}(-\Delta+a)^{p / 2} f \in L_{2}
$$

for all $\alpha$ with $|\alpha|<\lambda$. Here $a>\lambda^{1 / 2}$ and such $a$ is sufficiently large not to affect the exponential decay rate of $f$.

Theorem 6.4. (a) The convolution operator $\Gamma^{(2)}(x)$ is a continuous transformation from $H_{1, \lambda}$ to $H_{-1, \lambda}$ for any $\lambda \leqq \bar{m}$.

(b) If $\bar{m}<M$, then $\Gamma^{(2)}(x)$ is not densely defined, from $H_{1, \lambda}$ to $H_{-1, \lambda}$ for any $\lambda>\bar{m}$.

Remark. This is the precise sense in which $\Gamma^{(2)}(x)$ decays at the rate $\bar{m}$. Thus the presence of CDD zeros is equivalent to a decay rate $\bar{m}$ not associated with a physical particle.

Lemma 6.5. Let $T$ be a convolution operator with kernel $T(x)$, and suppose that when $a$ is sufficiently large,

$$
F(k)=\left(a+k^{2}\right)^{(q-p) / 2} \tilde{T}(k)
$$


is analytic for $k=\sigma+i \tau$, with $|\tau|<\lambda$, and is bounded in $\sigma$ for each fixed $|\tau|<\lambda$. Then the operator

$$
T: H_{p, \lambda} \rightarrow H_{q, \lambda}
$$

is continuous. (Here $|\tau|$ is the Euclidean norm.)

Proof. By Fourier transformation, $H_{p, \lambda}$ contains those functions $f$ such that for $|\alpha|<\lambda$ and $k$ real,

$$
e^{\alpha \cdot \nabla}\left(a+k^{2}\right)^{p / 2} \tilde{f}(k) \in L_{2} .
$$

Thus a convolution operator $T$ is bounded from $H_{p, \lambda}$ to $H_{q, \lambda}$ if for $k$ real,

$$
F_{\alpha} \equiv e^{\alpha \cdot \nabla}\left(a+k^{2}\right)^{(q-p) / 2} \tilde{T}(k) e^{-\alpha \cdot \nabla}
$$

is a bounded operator on $L_{2}$ for all real $\alpha,|\alpha|<\lambda$. In case the series converges, $F_{\alpha}$ is given by the Baker-Campbell-Hausdorff formula as a sum of multiplication operators on $L_{2}$,

$$
F_{\alpha}=\sum_{n=0}^{\infty} \frac{1}{n !}\left[(\alpha \cdot \nabla)^{n}\left(a+k^{2}\right)^{(q-p) / 2} \tilde{T}(k)\right] .
$$

Thus the operator norm of $F_{\alpha}$ on $L_{2}$ is bounded by

$$
\left\|F_{\alpha}\right\| \leqq \sum_{n=0}^{\infty} \frac{1}{n !}\left\|(\alpha \cdot \nabla)^{n}\left(a+k^{2}\right)^{(q-p) / 2} \tilde{T}_{(k)}\right\|_{L_{\infty}} .
$$

We estimate (6.11), using the Cauchy integral formula and the assumption of boundedness on $\left(a+k^{2}\right)^{(q-p) / 2} \tilde{T}(k)$. By a rotation of coordinates, we may suppose that $\alpha$ is parallel to the $x_{1}$ coordinate axis. Thus we estimate the derivatives of $\left(a+k^{2}\right)^{(q-p) / 2} \tilde{T}(k)$, for $k=\sigma$ real, by integrating over a disc $\left|\sigma_{1}-z_{1}\right| \leqq \beta$, centered at $\sigma$, and with $|\alpha|<\beta<\lambda$. Then

$$
\left|\left(\frac{\partial}{\partial k_{1}}\right)^{n}\left(a+k^{2}\right)^{(q-p) / 2} \tilde{T}(k)\right| \leqq A \beta^{-n}
$$

where $A=A(\beta)$ is independent of $n$ and $\sigma$. We conclude that

$$
\left\|F_{\alpha}\right\| \leqq A \sum_{n=0}^{\infty}|\alpha|^{n} \beta^{-n}<\infty .
$$

Proof of Theorem 6.4. We note

$$
-\tilde{\Gamma}^{(2)}(k)\left(k^{2}+m^{2}\right)^{-1}=\left[Z+\int_{a \geqq M^{2}}\left(\frac{k^{2}+m^{2}}{k^{2}+a}\right) d v(a)\right]^{-1},
$$

and we first establish $(b)$. If $\Gamma^{(2)}(x)$ has a decay rate $\beta$, the Fourier transform $\tilde{\Gamma}^{(2)}(k)$ has an analytic continuation defined for $|\tau|<\beta$. In particular, for $\sigma=0$,

$$
0 \neq Z+\int_{a \geqq M^{2}} \frac{m^{2}-\tau^{2}}{a-\tau^{2}} d v(a) .
$$

The integral in (6.13) decreases from 0 to

$$
\int \frac{m^{2}-M^{2}}{a-M^{2}} d v(a)<0
$$


as $\tau^{2}$ increases from $m^{2}$ to $M^{2}$. If $\bar{m}<M$, then $\bar{m}$ is the first zero of (6.13). Thus we have $\beta \leqq \bar{m}$, proving (b).

To establish (a), we note that for $A \leqq B, B+x>0$, the function $(A+x)(B+x)^{-1}$ is monotonic increasing in $x$. Thus for $k^{2}=(\sigma+i \tau)^{2}$,

$$
\begin{aligned}
\operatorname{Re}\left(\frac{k^{2}+m^{2}}{k^{2}+a}\right) & =\left.\frac{\left(\sigma_{1}^{2}-\tau^{2}+m^{2}\right)\left(\sigma_{1}^{2}-\tau^{2}+a\right)+4 \sigma_{2}^{2} \tau^{2}}{\left(\sigma_{1}^{2}-\tau^{2}+a\right)^{2}+4 \sigma_{2}^{2} \tau^{2}}\right|_{\sigma_{1}^{2}=\sigma_{2}^{2}=\sigma^{2}} \\
& \geqq \frac{\left(\sigma_{1}^{2}-\tau^{2}+m^{2}\right)\left(\sigma_{1}^{2}-\tau^{2}+a\right)}{\left(\sigma_{1}^{2}-\tau^{2}+a\right)^{2}}=\frac{\sigma_{1}^{2}-\tau^{2}+m^{2}}{\sigma_{1}^{2}-\tau^{2}+a} \\
& \geqq \frac{-\tau^{2}+m^{2}}{-\tau^{2}+a}
\end{aligned}
$$

For $|\tau|<\bar{m},(6.13)$ is strictly positive. Thus by (6.14),

$$
0<Z+\int_{a \geqq M^{2}} \frac{m^{2}-\tau^{2}}{a-\tau^{2}} d v(a) \leqq \operatorname{Re}\left[Z+\int \frac{k^{2}+m^{2}}{k^{2}+a} d v(a)\right] .
$$

We conclude that the right side of (6.12) is nonsingular and bounded away from zero uniformly in $\sigma$, for fixed $|\tau|<\bar{m}$. Furthermore in (6.12).

$$
\int_{a \geqq M^{2}} \frac{k^{2}+m^{2}}{k^{2}+a} d v(a)
$$

is analytic in $k^{2}$ for $\tau^{2}<\bar{m}^{2}$, since $\operatorname{Re}\left(k^{2}+a\right)=\sigma^{2}-\tau^{2}+a \geqq \sigma^{2}+M^{2}-\bar{m}^{2}>0$. Thus $\left(k^{2}+m^{2}\right)^{-1} \tilde{\Gamma}(k)$ is analytic $|\tau|<\bar{m}$ and bounded uniformly in $\sigma$ for fixed $|\tau|<\bar{m}$. We conclude that Lemma 6.5 applies to $\Gamma^{(2)}(x)$ in the case $p=-q=1$, $\lambda \leqq \bar{m}$, and the proof is complete.

\section{The Two Particle Bethe-Salpeter Kernel}

In this chapter, we prove the existence of the Bethe-Salpeter kernel $K_{\xi}$ in the Euclidean region, for almost every pure phase $\xi$. Let $R$ and $R_{0}$ be defined as in (1.6) so that

$$
R_{0}\left(x_{1}, x_{2}, y_{1}, y_{2}\right)=G_{\xi}^{(2)}\left(x_{1}, y_{1}\right) G_{\xi}^{(2)}\left(x_{2}, y_{2}\right)+G_{\xi}^{(2)}\left(x_{1}, y_{2}\right) G_{\xi}^{(2)}\left(x_{2}, y_{1}\right)
$$

and

$$
\begin{aligned}
R\left(x_{1}, x_{2}, y_{1}, y_{2}\right)= & R_{0}\left(x_{1}, x_{2}, y_{1}, y_{2}\right)+G_{\xi}^{(4)}\left(x_{1}, x_{2}, y_{1}, y_{2}\right) \\
& \int G_{\xi}^{(3)}\left(x_{1}, x_{2}, z\right) \Gamma_{\xi}^{(2)}\left(z, z^{\prime}\right) G_{\xi}^{(3)}\left(z^{\prime}, y_{1}, y_{2}\right) d z d z^{\prime} \\
= & \left\langle\phi\left(x_{1}\right) \phi\left(x_{2}\right)\left(I-P_{0}-P_{1}\right) \phi\left(y_{1}\right) \phi\left(y_{2}\right)\right\rangle
\end{aligned}
$$

Here $\Gamma_{\xi}^{(2)}=-G_{\xi}^{(2)-1}$, so that $R$ is one particle irreducible between the initial variables $x=\left(x_{1}, x_{2}\right)$ and the final variables $y=\left(y_{1}, y_{2}\right)$. Then $K_{\xi}$ is defined by

$$
R=R_{0}+R_{0} K_{\xi} R
$$

or

$$
K_{\xi}=R_{0}^{-1}-R^{-1}
$$


Theorem 7.1. Consider a $\mathscr{P}(\phi)_{2}$ model and suppose that (a) the $V=\infty$ limit is Lorentz invariant and (b) the mass $m=m_{\xi}$ in the pure phase $\xi$ is strictly positive. Then $K_{\xi}$ defined by (7.3)-(7.4) is a bounded operator from $\operatorname{Sym} H_{1}^{\otimes 2}$ to $H_{-1}^{\otimes 2}$.

Remark. We expect that the hypotheses are valid for all noncritical $\lambda \mathscr{P}(\phi)_{2}-\mu \phi$ models. They have been verified for weak coupling (i.e. $\lambda \ll m_{0}^{2}$ or $\mu \gg m_{0}^{2}$ ) $[25,26,45]$ and for even $\mathscr{P}(\phi)_{2}$ models in the single phase region or $\lambda \phi^{4}-\mu \phi$ with $\mu \neq 0$ $[39,28,9,44,29,24]$.

The kernel $K_{\xi}$ is formally two particle irreducible between initial and final states, so we expect a configuration exponential decay rate $3 m$, or $4 m$ for an even theory, assuming the absence of bound states and CDD zeros.

Proof. We know that $R_{0}^{-1}: H_{1}^{\otimes 2} \rightarrow H_{-1}^{\otimes 2}$ is bounded, from the spectral representation for the two point function [20]. To establish the same result for $R^{-1}$, we work in subspaces $\mathscr{H}_{ \pm}(P)$ of $H_{ \pm 1}^{\otimes 2}$ with definite total momentum, see (4.2). By Theorem $4.3(\mathrm{~b}), \mu_{\otimes n} G^{(4)}$ is Hilbert-Schmidt on $\mathscr{H}(P)$, so $G^{(4)}$ is Hilbert-Schmidt from $\mathscr{H}_{-}(P)$ to $\mathscr{H}_{+}(P)$. Since $\Gamma^{(2)}$ is an isomorphism of $H_{1}$ onto $H_{-1}$ [20], the last term in (7.2) is also Hilbert-Schmidt from $\mathscr{H}_{-}(P)$ to $\mathscr{H}_{+}(P)$, with norm 0 as $|P| \rightarrow \infty$. By the Fredholm alternative, $R^{-1}$ is defined if $R$ has no null vectors on $\mathscr{H}_{-}(P)$.

For simplicity, we suppose $\langle\phi\rangle_{\xi}=0$. In other words, we replace $\phi$ by $\hat{\phi}$ of Chapter 5. With this assumption, $G^{(2)}$ and $G^{(3)}$ are also Schwinger functions, and (7.2) becomes

$$
\begin{aligned}
R(x, y)= & S_{\xi}^{(4)}(x, y)-S_{\xi}^{(2)}\left(x_{1}, x_{2}\right) S_{\xi}^{(2)}\left(y_{1}, y_{2}\right) \\
& -S_{\xi}^{(3)}(x, z)\left[G^{(2)}\right]^{-1}\left(z, z^{\prime}\right) S_{\xi}^{(3)}\left(z^{\prime}, y\right) d z d z^{\prime}
\end{aligned}
$$

The above subtractions remove intermediate vacuum and one particle states, and are related to the projections $P_{0}$ and $P_{1}$ of Chapter 5. Let

$$
\chi(h)=\int \phi\left(x_{1}\right) \phi\left(x_{2}\right) h\left(x_{1}, x_{2}\right) d x_{1} d x_{2} .
$$

Then

$$
R(g \otimes h)=\left\langle\chi(g)\left(1-P_{0}-P_{1}\right) \chi(h)\right\rangle_{\xi} .
$$

Furthermore, representing $\left(1-P_{0}-P_{1}\right) \chi$ as a polynomial of degree 2 , we find

$$
\begin{aligned}
\left(1-P_{0}-P_{1}\right) \chi(h)= & \chi(h)-\langle\chi(h)\rangle_{\xi} \\
& +\int\langle\chi(h) \phi(z)\rangle_{\xi} \Gamma^{(2)}\left(z, z^{\prime}\right) \phi\left(z^{\prime}\right) d z d z^{\prime},
\end{aligned}
$$

and

$$
\langle\chi(h) \phi(z)\rangle_{\xi}=\int G^{(3)}\left(x_{1}, x_{2}, z\right) h\left(x_{1}, x_{2}\right) d x .
$$

We note

$$
\left.\left\langle|\chi(h)-\langle\chi\rangle|^{2}\right\rangle_{\xi}=\int G^{(4)}-R_{0}\right) h^{-}(x) h(y) d x d y .
$$

We now consider $R$ acting from $\mathscr{H}_{-}(P)$ to $\mathscr{H}(P)$, and we suppose that $R$ has a nonzero null space. For simplicity, we treat the case $P=0$. We suppose that there is a nonzero even function $f$ in $L_{2}\left(R^{2}\right)$ such that

$$
0=\int\left(\mu_{\otimes} R\right)(x) f\left(x_{1}-x_{2}\right)^{-} f\left(x_{3}-x_{4}\right) d x_{2} d x_{3} d x_{4} .
$$


We multiply this equation by

$$
\int g\left(x_{1}+x_{2}-w\right)^{-} g\left(x_{3}+x_{4}+w\right) d w=g^{-} * g\left(x_{1}+x_{2}+x_{3}+x_{4}\right)
$$

and integrate over $x_{1}$. For $g \in C_{0}^{\infty}$, we obtain

$$
0=\int\left(\mu_{\otimes} R\right)(x) h\left(x_{1}, x_{2}\right)^{-} h\left(x_{3}+w, x_{4}+w\right) d x d w,
$$

where

$$
h(y, z)=f(y-z) g(y+z) .
$$

In terms of (7.6)-7.9),

$$
0=\int d w\left\langle\chi(h),\left(I-P_{0}-P_{1}\right) \chi\left(h_{w}\right)\right\rangle_{\xi},
$$

where $h_{w}(y, z)=h(y+w, z+w)$. By Theorem 5.8 and Remark 5.9, with $X(h)=B=C$, we have

$$
0=\int h(y+a, z+a) d a=\int f(y-z) g(y+z+2 a) d a=\text { const. } f(y-z) .
$$

Thus $\chi(h)=0$ and so $R$ has no null vectors (except zero) in the space of definite momentum.

It follows that $R \uparrow \mathscr{H}_{-}(P)$ has a bounded inverse. Since the inverse depends continuously on $P$, and remains bounded uniformly as $P \rightarrow \infty, R$, defined on $\mathscr{H}_{-1}$ has a bounded inverse. This completes the proof of Theorem 7.1.

\section{Three Particle Equations}

The three particle scattering equation is defined in analogy to Chapter 7 , after subtraction of the two body rescattering processes. We give two procedures for these subtractions, one based on the two-body Bethe-Salpeter kernel, the other based on Faddeev-type equations for two-body rescattering processes. We expect that a quantitative analysis of the three-body equation will yield insight into such problems as: Do three-particle bound states occur? Is $S$ unitary up to the four-particle threshold?

Let $\alpha=12,13,23$ labeled pairs of initial or final particles. A two particle process occurs in a channel labeled by a pair $\alpha, \beta$. The free Green's function for a two particle channel is

$$
R_{0}^{\alpha \beta}=G^{(2)}\left(x_{i}, y_{j}\right)\left[G^{(2)}\left(x_{\alpha_{1}}, y_{\beta_{1}}\right) G^{(2)}\left(x_{\alpha_{2}}, y_{\beta_{2}}\right)+G^{(2)}\left(x_{\alpha_{1}}, y_{\beta_{2}}\right) G^{(2)}\left(x_{\alpha_{2}}, y_{\beta_{1}}\right)\right]
$$

where $i \notin \alpha, j \notin \beta$. The total $R_{0}$ is

$$
R_{0}=\sum_{\beta} R_{0}^{\alpha \beta=} \sum_{\pi} G^{(2)}\left(x_{1}-y_{i}\right) G^{(2)}\left(x_{2}-y_{j}\right) G^{(2)}\left(x_{3}-y_{k}\right),
$$

where $\sum_{\pi}$ ranges over the six permutations $(i, j, k)$ of $(1,2,3)$.

We also define the two particle scattering kernels

$$
R_{\alpha \beta}=G^{(2)}\left(x_{i}, y_{j}\right) G^{(4)}\left(x_{\alpha}, y_{\beta}\right),
$$

where $i \notin \alpha, j \notin \beta$. Graphically, $R_{\alpha \beta}$ represents scattering processes with initial state $(i, \alpha)$, final state $(j, \beta)$, and two particle scattering in the $\alpha, \beta$ channel. See Fig. 8.1. 


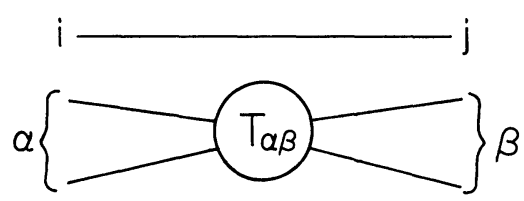

Fig. 8.1. A two particle process

Proposition 8.1. The operators $R_{0}^{\alpha \beta}, R_{0}$, and $R_{\alpha \beta}$ are bounded transformations $H_{-1}^{\otimes 3}$ to $H_{1}^{\otimes 3}$. The norm of $R_{\alpha \beta}$ is $O(\lambda)$ as $\lambda \equiv \sup \left|\lambda_{j}\right| \rightarrow 0$.

Proof. This follows immediately by the bounds of [20].

Definition 8.2. The two-body Bethe-Salpeter kernel $K_{\alpha \alpha}$ for the three body problem is

$$
\left.K_{\alpha \alpha}=R_{0}^{\alpha \alpha}\right)^{-1}-\left(R_{0}^{\alpha \alpha}+R_{\alpha \alpha}\right)^{-1} .
$$

$K_{\alpha \beta}$ is defined by a permutation of the $y$ variables of $K_{\alpha \alpha}$. The two body amplitude $R_{2 \text {-body }}$ satisfies

$$
R_{2 \text {-body }}=R_{0}+\frac{1}{9} R_{0} \sum_{\alpha, \beta} K_{\alpha \beta} R_{2 \text {-body }} .
$$

We remark that $K_{\alpha \beta}$ has the form

$$
K_{\alpha \beta}=K \otimes(-\Gamma),
$$

where $K$ is the Bethe-Salpeter kernel (7.4) for the channel $(\alpha \beta)$ and $-\Gamma$ is the inverse propagator for the remaining particle.

Theorem 8.3. Under the hypotheses of Theorem 7.1, $K_{\alpha \beta}$ and

$$
R_{2 \text {-body }}^{-1}=R_{0}^{-1}-\frac{1}{9} \sum_{\alpha, \beta} K_{\alpha \beta}
$$

are bounded maps from $\operatorname{Sym} H_{1}^{\otimes 3}$ to $H_{-1}^{\otimes 3}$, with $\left\|K_{\alpha \beta}\right\| \leqq O(\lambda)$ as $\lambda \rightarrow 0$. Also for $\lambda$ sufficiently small, $R_{2 \text {-body }}$ is a bounded map from $\operatorname{Sym} H_{-1}^{\otimes 3}$ to $H_{1}^{\otimes 3}$.

Proof. The bound on $K_{\alpha \beta}$ follows by Theorem 7.1 which bounds $K$ of (7.4) and the properties of $\Gamma$ established in [20]. Since $R_{0}^{-1}$ is bounded from Sym $H_{1}^{\otimes 3}$ to $H_{-1}^{\otimes 3}$, the existence and boundedness of $R_{2 \text {-body }}^{-1}$ follows. We note by (8.5),

$$
\left(I-\frac{1}{9} R_{0} \sum K_{\alpha \beta}\right) R_{2 \text {-body }}=R_{0},
$$

and, by the above, the norm of $R_{0} K_{\alpha \beta}$ from $H_{1}^{\otimes 3}$ to $H_{1}^{\otimes 3}$ is $O(\lambda)$. Thus for $\lambda$ sufficiently small,

$$
\left(I-\frac{1}{9} R_{0} \sum K_{\alpha \beta}\right)^{-1}: H_{1}^{\otimes 3} \rightarrow H_{1}^{\otimes 3}
$$

is bounded. It follows that

$$
R_{2 \text {-body }}: H_{-1}^{\otimes 3} \rightarrow H_{1}^{\otimes 3}
$$

exists and is bounded.

Theorem 8.4. Consider $R_{2 \text {-body }}^{-1}$ restricted to a constant momentum subspace:

$$
R_{2-\text { body }}^{-1}(P): \operatorname{Sym}_{H_{+}}(P) \rightarrow \mathscr{H}_{-}(P) \text {. }
$$


Then for $|P|$ sufficiently large, $R_{2 \text {-body }}(P)$ is bounded from $\operatorname{Sym} \mathscr{H}_{-}(P)$ to $\mathscr{H}_{+}(P)$. (Here the minimum $|P|$ may depend on $\lambda$.) Also as $|P| \rightarrow \infty$,

$$
\left\|R_{2 \text {-body }}(P)-R_{0}(P)\right\|_{-,+} \rightarrow 0 \text {. }
$$

Proof. Each of the operators $R_{0}, R_{0}^{\alpha \beta}, R_{\alpha \beta}, K_{\alpha \beta}$ is translation invariant, and thus acts on constant momentum subspaces. By Theorem $4.3(\mathrm{~b}), R_{\alpha \beta}\left(R_{0}^{\alpha \beta}\right)^{-1}$ is a bounded operator on $\operatorname{Sym} \mathscr{H}_{+}(P)$, with norm $o(1)$ as $|P| \rightarrow \infty$. We choose $|P|$ sufficiently large so that

$$
R_{0} K_{\alpha \beta}=-R_{0}\left(R_{0}^{\alpha \beta}\right)^{-1} \sum_{n=1}^{\infty}\left(-R_{\alpha \beta}\left(R_{0}^{\alpha \beta}\right)^{-1}\right)^{n}
$$

has norm on $\operatorname{sym} \mathscr{H}_{+}(P)$ less than 1 . Thus $\left\|\sum_{\alpha \beta} \frac{1}{9} R_{0} K_{\alpha \beta}\right\|<1$ on sym $\mathscr{H}_{+}(P)$ and

$$
R_{2 \text {-body }}^{-1}=R_{0}^{-1}\left(1-\frac{1}{9} \sum_{\alpha, \beta} R_{0} K_{\alpha \beta}\right)
$$

is invertible. The inverse $R_{2-\text { body }}(P)$ is bounded from $\operatorname{Sym} \mathscr{H}_{-}(P)$ to $\mathscr{H}_{+}(P)$, since $R_{0}(P)$ is. Also $\left(R_{0} K_{\alpha \beta}\right)(P)$ has norm $o(1)$ as $|P| \rightarrow \infty$, so $\left\|R_{2 \text {-body }}(P)-R_{0}(P)\right\|_{-,-}$is $o(1)$. Here $\|\cdot\|_{-}+$denotes the norm from sym $\mathscr{H}_{-}(P)$ to $\mathscr{H}_{+}(P)$.

We remark that instead of using the direct definition (8.5) of $R_{2 \text {-body }}$, we could also use Faddeev type equations to separate the two body forces. Let $R(\alpha)$ denote the sum of 2-body rescattering diagrams in which the initial two-body scattering involves the pair $\alpha$. Then the Feddeev equations are

$$
\begin{aligned}
R(\alpha)= & R_{0}^{\alpha \alpha}+R_{\alpha \alpha} \\
& +\sum_{\beta \neq \alpha} R_{\alpha \beta}\left(R_{0}^{\beta \beta}+R_{\beta \beta}\right)^{-1} R(\beta) \\
= & R_{0}^{\alpha \alpha}+R_{\alpha \alpha} \\
& \sum_{\beta \neq \alpha} R_{\alpha \beta}\left[\left(R_{0}^{\beta \beta}\right)^{-1}-K_{\beta \beta}\right] R(\beta) .
\end{aligned}
$$

As above, we find

Theorem 8.5. For $\lambda$ sufficiently small, the Eq. (8.7) have a unique solution $R(\alpha): H_{-1}^{\otimes 3} \rightarrow H_{1}^{\otimes 3}$. For constant total momentum $P$, the equations (8.7) have a unique solution $R(\alpha, P): \mathscr{H}_{-}(P) \rightarrow \mathscr{H}_{+}(P)$, for $|P|$ sufficiently large (depending on $\lambda$ ).

For small $\lambda$, we define

$$
\bar{R}_{2 \text {-body }}=\sum_{\alpha}\left(R(\alpha)-R_{0}^{\alpha \alpha}\right)+R_{0} .
$$

We expect that $R_{2 \text {-body }}=\bar{R}_{2 \text {-body }}$.

We now define the three body Bethe-Salpeter kernel $K^{(3)}$, by the equations

$$
R^{(3)}\left(x_{1}, x_{2}, x_{3}, y_{1}, y_{2}, y_{3}\right)=\left\langle\prod_{i=1}^{3} \phi\left(x_{i}\right)\left(I-\sum_{j=0}^{2} P_{j}\right) \prod_{k=1}^{3} \phi\left(x_{6}\right)\right\rangle,
$$

and

$$
R^{(3)-1}=R_{2-\text { body }}^{-1}-K^{(3)}
$$

or

$$
R^{(3)}=R_{2 \text {-body }}+R_{2 \text {-body }} K^{(3)} R^{(3)} \text {. }
$$

Theorem 8.6. Under the hypothesis of Theorem 7.1, $R^{(3)^{-1}}$ and $K^{(3)}$ exist as bounded operators from Sym $H_{1}^{\otimes 3}$ to $H_{-1}^{\otimes 3}$, and satisfy (8.10). For weak coupling, (8.11) also holds. 
Remark. $N$-body equations, as well as a further study of the three body equations, will be contained in a subsequent article. By definition $-K^{(n)}$ is the connected part of $R^{(n)^{-1}}$.

\section{References}

1. Araki, H.: On the algebra of all local observables. Prog. Theor. Phys. 32, 844-854 (1964)

2. Bratteli, O.: Conservation of estimates in quantum field theory. Comm. Pure and Appl. Math. 25, 759-779 (1972)

3. Bros, J.: Some analyticity properties implied by the two particle structure of Green's functions in general quantum field theory. In: Gilbert, R., Newton, R. (Eds.): Analytic methods in mathematical physics. New York: Gordon and Breach 1970

4. Castillejo, L., Dalitz, R., Dyson, F.: Low's scattering equation for the charged and neutral scalar theories. Phys. Rev. 101, 453-458 (1956)

5. Dobrushyn, R., Minlos, R.: Construction of a one dimensional quantum field via a continuous Markov field. Funct. Anal. and its Appl. 7, 324-325 (1973) (English transl.)

6. Eckmann, J.-P. Magnen, J., Seneor, R.: Decay properties and Borel summability for the Schwinger functions in $P(\phi)_{2}$ theories. Commun. math. Phys. 39, 251-271 (1975)

7. Faris, W., Lavine, R.: Commutators and self-adjointness of Hamiltonian operators. Commun. math. Phys. 35, 39-48 (1974)

8. Feldman, J.: On the absence of bound states in the $\phi^{4}$ quantum field model without symmetry breaking. Canad. J. Phys. 52, 1583-1587 (1974)

9. Fröhlich, J.: Verification of axioms for Euclidean and relativistic fields and Haag's theorem in a class of $P(\phi)_{2}$ models. Ann. Inst. H. Poincaré (to appear)

10. Fröhlich, J.: Schwinger functions and their generating functionals. I. Helv. Phys. Acta (to appear)

11. Fröhlich, J.: Schwinger functions and their generating functionals. II. Adv. Math. (to appear)

12. Ginibre, J.: General formulation of Griffiths' inequalities. Comm. Math. Phys. 16, 310-328 (1970)

13. Glimm, J., Jaffe, A.: A $\lambda \phi^{4}$ quantum field theory without cutoffs II, the field operators and the approximate vacuum. Ann. of Math. 91, 362-401 (1970)

14. Glimm, J., Jaffe, A.: The $\left(\lambda \phi^{4}\right)_{2}$ quantum field theory without cutoffs. III. The physical vacuum. Acta Math. 125, 203-261 (1970)

15. Glimm, J., Jaffe, A.: The $\left(\lambda \phi^{4}\right)_{2}$ quantum field theory without cutoffs. IV. Perturbations of the Hamiltonian. J. Math. Phys. 13, 1558-1584 (1972)

16. Glimm, J., Jaffe, A.: The energy momentum spectrum and vacuum espectation values in quantum field theory. J. Math. Phys. 11, 3335-3338 (1970)

17. Glimm, J., Jaffe, A.: The energy momentum spectrum and vacuum expectation values in quantum field theory. II. Commun. math. Phys. 22, 1-22 (1971)

18. Glimm, J., Jaffe, A.: Boson quantum field models. In: Streater, R. (Ed.): Mathematics of contemporary physics. New York: Academic Press 1972

19. Glimm, J., Jaffe, A. : Positivity of the $\phi_{3}^{4}$ Hamiltonian. Fortschritte der Physik 21, 327-376 (1973)

20. Glimm, J., Jaffe, A.: Entropy principle for vertex functions in quantum field models. Ann. Inst. H. Poincaré 21, 1-26 (1974)

21. Glimm, J., Jaffe, A.: $\phi_{2}^{4}$ quantum field model in the single phase region: Differentiability of the mass and bounds on critical exponents. Phys. Rev. D 10, 536-539 (1974)

22. Glimm, J., Jaffe, A.: A remark on the existence of $\phi_{4}^{4}$. Phys. Rev. Lett. 33, $440-442$ (1974)

23. Glimm,J., Jaffe, A.: Absolute bounds on vertices and couplings. Ann. 1'Inst. H. Poincaré 22 (to appear)

24. Glimm,J., Jaffe, A.: On the approach to the critical point. Ann. l'Inst. H. Poincare 22 (to appear)

25. Glimm, J., Jaffe, A., Spencer,T.: The particle structure of the weakly coupled $P(\phi)_{2}$ model and other applications of high temperature expansions. In: Velo, G., Wightman, A. (Eds.): Constructive quantum field theory. Lecture notes in Physics 25. Berlin Heidelberg New York: Springer 1973

26. Glimm,J., Jaffe, A., Spencer, T.: The Wightman axioms and particle structure in the $P(\phi)_{2}$ quantum field model. Ann. Math. 100, 585-632 (1974)

27. Guerra,F., Rosen,L., Simon, B.: Nelson's symmetry and the infinite volume behavior of the vacuum in $P(\phi)_{2}$. Commun. math. Phys. 27, 10-22 (1972) 
28. Guerra, F., Rosen,L., Simon, B.: The $P(\phi)_{2}$ Euclidean quantum field theory as çlassical statistical mechanics. Ann. Math. 101, 111-259 (1975)

29. Guerra, F., Rosen, L., Simon, B.: Correlation inequalities and the mass gap. III. Mass gap for a class of strongly coupled theories with nonzero external field. Commun. math. Phys., to appear

30. Jost, R.: The general theory of quantized fields. American Mathematical Society, Providence, 1965

31. Lebowitz, J., Penrose, O.: Analytic and clustering properties of the thermodynamic functions and distribution functions for classical lattice and continuum systems 11, 99-124 (1968)

32. Lebowitz, J., Penrose, O.: Decay of correlations. Phys. Rev. Lett. 31, 749-752 (1973)

33. McBryan, O.: Local generators for the Lorentz group in the $P(\phi)_{2}$ model. Nuovo Cimento, $18 \mathrm{~A}$, $654-662(1973)$

34. McBryan, O.: Vector currents in the Yukawa ${ }_{2}$ quantum field theory (preprint)

35. McBryan, O.: Self adjointness of relatively bounded quadratic forms and operators. J. Funct. Anal. (to appear)

36. McBryan, O., Park, Y.: Lorentz covariance of the Yukawa ${ }_{2}$ quantum field theory. J. Math. Phys. 16, 105-110 (1975)

37. Nelson, E.: Time ordered operator products of sharp time quadratic forms. J. Funct. Anal. 11, $211-219(1972)$

38. Nelson, E.: The construction of quantum fields from Markov fields. J. Funct. Anal. 12, 97-112 (1973)

39. Nelson, E.: Probability theory and Euclidean field theory. In: Velo, G., Wightman, A. (Eds.): Constructive quantum field theory. Lecture notes in Physics 25. Berlin Heidelberg New York: Springer 1973

40. Osterwalder, K., Schrader, R.: Axioms for Euclidean Green's functions. Commun. math. Phys. 31, 83-112 (1974)

41. Osterwalder,K., Schrader, R.: Axioms for Euclidean Green's functions. II. Commun. math. Phys. 42, 281-305 (1975)

42. Rosen, L.: A $\lambda \phi^{2 n}$ field theory without cutoffs. Commun. math. Phys. 16, 157-183 (1970)

43. Schrader, R.: Yukawa quantum field theory in two space-time dimensions without cutoff. Ann. Phys. 70, 412-457 (1972)

44. Simon, B.: The $P(\phi)_{2}$ Euclidean quantum field theory. Princeton: Princeton University Press 1974

45. Spencer, T.: The mass gap for the $P(\phi)_{2}$ quantum field model with a strong external field. Commun. math. Phys. 39, 63-76 (1974)

46. Spencer, T.: The absence of even bound states in $\phi_{2}^{4}$. Commun. math. Phys. 39, 77-79 (1974)

47. Spencer, T.: The decay of the Bethe Salpeter kernel in $P(\phi)_{2}$ quantum field models. Commun. math. Phys. (to appear)

48. Spencer, T., Zirilli, F.: Private communication

49. Symanzik, K.: A modified model for Euclidean quantum field theory. N.Y.U. (preprint) 1964

50. Tucciarone, A.: A relativistic treatment of the three body problem. Nuovo Cimento 41 A, 204-221 (1966)

51. Glimm, J., Jaffe, A.: $\phi^{J}$ bounds in $P(\phi)_{2}$ quantum field models. Proceedings of the International Colloquium on Mathematical Methods in Quantum Field Theory, Marseille, 1975

Communicated by A. S. Wightman 